\title{
Closed-loop field development with multipoint geostatistics and statistical performance assessment
}

\author{
Mehrdad G Shirangi*
}

April 3, 2019

Keywords: Optimization under uncertainty, Closed-loop optimization, Massive computational experiment, Performance assessment, Data assimilation, Spatial statistics, Subsurface flow, Well placement, Mixed-integer optimization, Principal component analysis.

\begin{abstract}
Closed-loop field development (CLFD) optimization is a comprehensive framework for optimal development of subsurface resources. CLFD involves three major steps: 1) optimization of full development plan based on current set of models, 2) drilling new wells and collecting new spatial and temporal (production) data, 3) model calibration based on all data. This process is repeated until the optimal number of wells is drilled. This work introduces a new CLFD implementation for complex systems described by multipoint geostatistics (MPS). Model calibration is accomplished in two steps: conditioning to spatial data by a geostatistical simulation method, and conditioning to production data by optimization-based PCA. A statistical procedure (TruMAP) is presented to assess the performance of CLFD. For performance assessment by TruMAP, the methodology is applied to an oil reservoir example for 25 different true-model cases. Application of a single-step of CLFD, improved the true NPV in $64 \%-80 \%$ of cases. The full CLFD procedure (with three steps) improved the true NPV in $96 \%$ of cases, with an average improvement of $37 \%$. These results indicate the effectiveness of performing multiple steps of closed-loop optimization. This massive computational experiment involved about 9.5 million reservoir simulation runs that took about 320,000 CPU hours.
\end{abstract}

\section{Introduction}

For optimal operation of oil and gas resources, engineers typically build physics-based flow simulation models. These models need to be calibrated with observed spatial and temporal data so that they can be reliable for purposes such as optimization and decision making. The common approach for management of these systems is closed-loop optimization which involves three major steps, repeated throughout the project life: 1) optimization of decision

*Stanford University, Stanford, CA 94305, United States. Email addresses: mehr@stanford.edu, Mehrdad.GharibShirangi@ge.com 
parameters, 2) implementation and operation for a time period, 3) and model calibration for consistency with (new) observed data. For different types of reservoir problems, various approaches have been proposed for Steps 1 and 3, i.e., the optimization and the model calibration step. In addition, implementations of closed-loop optimization may differ on type of decision parameters included in these two steps.

In the context of conventional oil reservoir management, decision parameters depend on the recovery process employed. The most common recovery process is water-flooding where water is injected through injection wells to push the oil towards producer wells. There are other processes such as gas injection, water-alternating-gas injection, surfactant and polymer flooding, and steam-assisted gravity drainage (SAGD) which are employed based on subsurface reservoir condition and economic parameters (e.g., availability/cost of surface facilities). This work focuses on the water-flooding process, though the framework and results can be extended to other recovery processes. Decision parameters, in this context, include the number, type (oil production/water injection), location, and controls (time-varying well bottom-hole pressures) of new wells throughout the project life.

Closed-loop field development (CLFD) optimization, introduced by Shirangi and Durlofsky (2015), is a comprehensive reservoir management framework. In the optimization step of CLFD, the number, type, location and controls of new wells (together with controls of the existing wells) are optimized. The model calibration step of CLFD involves integration/assimilation of new spatial data (hard data from new wells) and temporal data (production data from existing wells). The impact of CLFD research is significant as drilling new wells is one of the most expensive parts of reservoir operations. In Shirangi and Durlofsky (2015), the CLFD framework is presented with two-point geostatistical models. In recent years, however, there has been significant research on multipoint geostatistics (MPS) which is able to model complex geological features such as channels and deltaic fans.

In this work, a closed-loop field development framework is developed and applied for reservoir models described by multipoint geostatistics. A two-step model calibration procedure is presented where we employ an efficient geostatistical algorithm to integrate spatial data, and a recent model calibration algorithm for integration of temporal data (production history). A statistical assessment procedure is then presented for performance evaluation of this closed-loop framework. In this assessment, the project outcome (true NPV) is treated as a random variable and the improvement in true NPV is investigated after each CLFD step.

Before the introduction of CLFD framework, most of the reservoir optimization research has focused on developing improved approaches for optimal operation of existing wells, referred to as closed-loop reservoir management (CLRM). Compared to CLFD, CLRM does not involve drilling new wells. In optimization step of CLRM, referred to as production optimization or well control optimization, continuous operational settings of existing wells are optimized. The model calibration of CLRM only includes new temporal data, and integration of new spatial data is not typically considered. CLRM has been investigated extensively (see, e.g., Jansen et al $(2009,2005)$ ). Most papers on CLRM investigated the application of particular model calibration and optimization approaches for water-flooding operations (e.g., Aitokhuehi and Durlofsky (2005); Chen et al (2009)). CLRM has also been applied to SAGD operations (Regtien, 2010), and for the management of geological carbon storage operations (Cameron and Durlofsky, 2014). It's worth mentioning that Jansen et al 
(2005, 2009) envisioned the use of optimal position of sidetracks or infill wells in closed-loop optimization, which is now performed in CLFD.

In the field development planning (FDP) problem, encountered in CLFD, decision parameters may include the number of new wells, well type (producer or injector), well locations, drilling sequence, and well settings. Most papers on FDP considered the optimization of only a subset of these parameters, mainly the location of new wells (referred to as the well placement problem). Many of the methods developed for this problem entail stochastic (global) search methods such as GAs (Ozdogan and Horne, 2006), PSO (Onwunalu and Durlofsky, 2010; Arnold et al., 2016), evolution strategy with covariance matrix adaptation (CMA-ES) (Bayer and Finkel, 2007; Bayer et al, 2008, Bouzarkouna et al, 2012), and differential evolution (DE) (Bayer et al, 2010; Nwankwor et al, 2013). Local optimization methods such as SPSA (Shirangi and Mukerji, 2012), and pattern search techniques (Wilson and Durlofsky, 2013; Cameron and Durlofsky, 2012) have also been applied for well placement optimization.

The more complex problem of jointly optimizing various parameters in FDP has also been investigated. Bellout et al (2012); Li and Jafarpour (2012); Zhang et al (2017) presented different algorithms for the joint optimization of well location and controls. Isebor et al (2014a b) developed a formulation based on a hybrid of PSO, a global stochastic search algorithm, and mesh adaptive direct search (MADS), a local pattern search method. This PSO-MADS procedure can simultaneously optimize the number and type (e.g., injector or producer) of new wells and the drilling sequence, in addition to well locations and controls. This algorithm will be used in this work for the optimization step.

Model calibration is the third major step in CLFD. In model calibration (also referred to as history matching, or data assimilation), the goal is to learn from data through generating one or more computational models that are consistent with prior geological information and new spatial data, and provide flow simulation results that closely match observed (temporal) production data. The model calibration methods typically involve a challenging minimization problem as this problem is usually ill-posed and the number of unknown model parameters can be very large. The ill-posedness of model calibration can be mitigated by reducing the number of parameters through an appropriate parameterization such as TSVD (Shirangi, 2011, 2014, Shirangi and Emerick, 2016; Bjarkason et al, 2017; Dickstein et al, 2017), ensemble-based methods (Rafiee and Reynolds, 2017; Rafiee, 2017; Rafiee and Reynolds, 2018), and PCA (Vo and Durlofsky, 2016). Vo and Durlofsky (2015, 2014) presented a differentiable PCA-based parameterization (O-PCA) that enables application of efficient gradient-based approaches for model calibration of complex channelized systems. This optimization-based principal component analysis (O-PCA) approach is used in the CLFD model calibration step in this work.

The CLFD framework has also been applied in recent work by other researchers. Morosov and Schiozer (2016) applied the CLFD to a realistic example, while Hanea et al (2017) applied the CLFD framework in simpler settings where only drilling sequence is optimized. Compared with the original work (Shirangi and Durlofsky, 2015; Shirangi, 2013), they used different reservoir modeling and different model calibration and optimization approaches in their CLFD application. Both papers, reported that CLFD significantly improved truemodel NPV in most cases; however, in at least one case in each work, the true-model NPV decreased slightly after the application of CLFD. More recently, Hidalgo et al (2017) applied 
the CLFD algorithm to a realistic reservoir case and reported $40.8 \%$ improvement in (true) NPV.

In Shirangi and Durlofsky (2015), reservoir properties were represented in terms of twopoint spatial statistics. The first contribution of this work is to present and apply a CLFD implementation for complex channelized models, described by a training image. A training image contains a range of valuable information such as orientation, proportions, affinity, trend that need to be reproduced (Tahmasebi et al, 2014; Mariethoz and Caers, 2014). This extension involves particular treatments for integration of spatial and temporal data in the model calibration step. This new implementation is to improve CLFD model calibration step by utilizing a multipoint geostistical algorithm within the closed-loop optimization workflow. The second contribution of this work is to present and apply the TruMAP (true-model based assessment of performance) procedure. The goal here is to statistically characterize the performance of a closed-loop optimization algorithm in terms of improvement in true NPV.

This paper proceeds as follow. In the next section, the CLFD framework and treatments for the model calibration and the optimization step are described. In the section after that, computational results for CLFD application are presented for a two-dimensional binary channelized reservoir case. Results of statistical assessment of CLFD performance is then presented in this section. Finally, conclusions and suggestions for future work are provided.

\section{Methodology: closed-loop field development optimization}

Closed-loop field development (CLFD) (Fig. 1) has three major steps as follows. 1) Optimization: optimize the "full development plan" (the number, type, location and controls of all new wells together with controls of existing wells), for a set of models describing the current state of knowledge, 2) operation: drill and complete $n_{\text {rig }}$ new wells, produce from existing wells, and collect new spatial and temporal data, 3) model calibration (history matching): update models for consistency with new data. These steps are repeated until drilling additional wells does not (sufficiently) increase the expected NPV, at which step, the CLRM workflow is applied. We now describe the CLFD workflow in more detail.

We consider the oil field development optimization problem where decision parameters of new wells are determined. In practical settings, the number of drilling rigs is limited and new wells are drilled sequentially, one or a few at a time. We let $n_{\text {rig }}$ denote the number of rigs (in this work, we set $n_{\text {rig }}=2$ ), and we let $T_{\text {drill }}$ denote the duration (in days) that it takes to drill and complete a new well and start production/injection. The initial field development plan is determined by optimizing the (full) development plan over a prior set of geological models, generated by use of a geostatistical simulation method. We let $t_{i}$, $i \geq 0$, denote the time that the $(i+1)$ th group of wells is drilled. The first group of wells is drilled at $t_{0}=0$.

After drilling and completion of each group of $n_{\text {rig }}$ new wells, new spatial data (hard data from well locations) and new temporal data (production data) are collected. The CLFD model calibration is performed at each $t_{i}(i \geq 1)$ to update models for consistency with all data. Here, observed hard data corresponds to the rock property value at (new and existing) well location(s) in the true-model (without any noise added), and is integrated through a geostatistical algorithm. Synthetic observed production data (for model calibration) is 


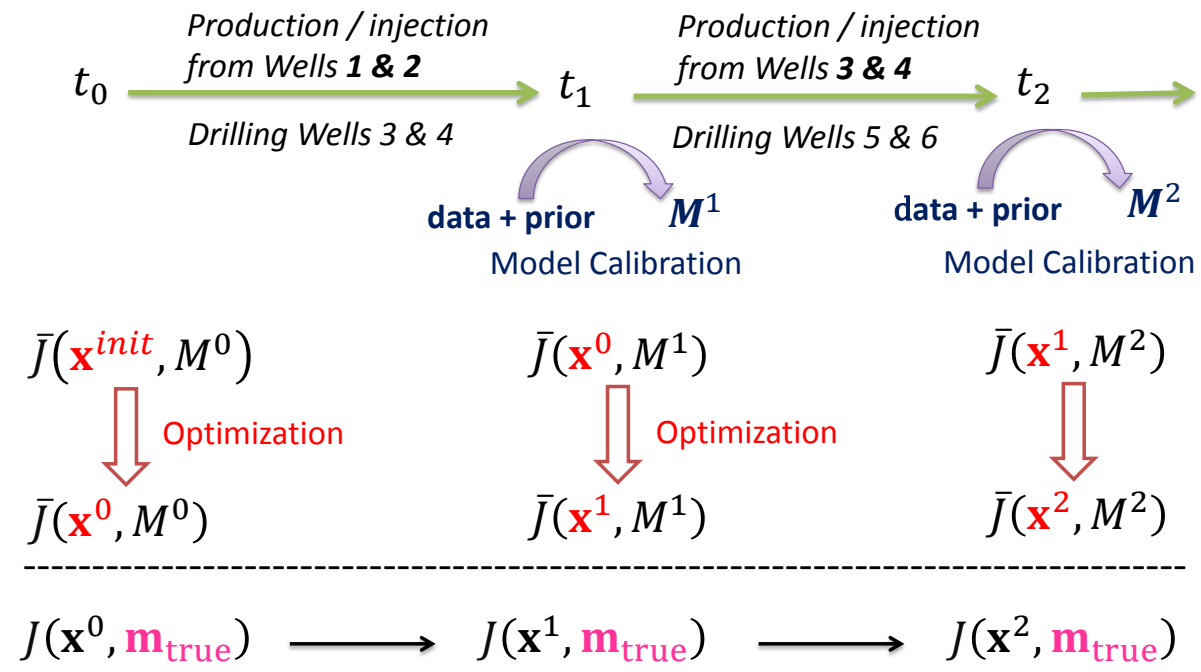

Figure 1: Schematic and notations for CLFD framework with $n_{\text {rig }}=2$.

generated by simulating the true-model and adding random noise to the true data. The true-model here is a realization randomly selected from the prior set.

The optimization is then performed to determine decision parameters for all future wells (those to be delivered at $t_{i+1}$ and later steps), and controls of existing wells. Note that only the controls after $t_{i}$ are optimized here. Optimizing the full development plan at each step is to avoid a greedy approach where one would only optimize the next $n_{\text {rig }}$ wells by either freezing or not considering later wells in the project horizon. A comparison between greedy (well-by-well) optimization and optimization of full development plan is discussed in Example 1 in Shirangi and Durlofsky (2015). CLFD workflow is shown in Fig. 1. The optimization and model calibration steps (together with notations) are discussed next, in turn.

\subsection{CLFD optimization step}

In CLFD, we use $M^{i}=\left\{\mathbf{m}_{1}^{i}, \ldots \mathbf{m}_{N_{R}}^{i}\right\}$, a set of $N_{R}$ models, to represent the (current) geological knowledge at step $i$. At each CLFD optimization step, the expected net present value (NPV) of the project is optimized for a set of representative models selected from the full set. The problem is formulated as

$$
\begin{aligned}
& \operatorname{maximize} \bar{J}\left(\mathbf{x}, M_{\text {rep }}^{i}\right), \\
& \text { subject to } \mathbf{x}^{1} \leq \mathbf{x} \leq \mathbf{x}^{\mathrm{u}}, \quad g_{k}(\mathbf{x}) \leq 0,0 \leq k \leq n_{\text {ie }},
\end{aligned}
$$

where $\mathbf{x}$ is the vector of decision parameters, $M_{\text {rep }}^{i}=\left\{\mathbf{m}_{r_{1}}^{i}, \ldots \mathbf{m}_{r_{n_{r}}}^{i}\right\}$ is a set of $n_{r}$ representative models $\left(M_{\mathrm{rep}}^{i}\right.$ is a subset of $\left.M^{i}\right), \mathrm{x}^{\mathrm{l}}$ and $\mathrm{x}^{\mathrm{u}}$ denote the lower and upper bound vectors, $n_{\mathrm{ie}}$ is the number of inequality constraints, and $\bar{J}$ is the expected NPV defined as

$$
\bar{J}\left(\mathbf{x}, M_{\mathrm{rep}}\right)=\frac{1}{n_{r}} \sum_{f=1}^{n_{r}} J\left(\mathbf{x}, \mathbf{m}_{f}\right) .
$$


Computing NPV value for each model $f$ involves a flow simulation run. In this work, the water-flooding process with two-phase flow is considered where NPV is computed as

$$
\begin{aligned}
J\left(\mathbf{x}, \mathbf{m}_{f}\right) & =\sum_{l=1}^{N_{l}}\left[\sum_{k=1}^{N_{P}}\left(p_{\mathrm{o}} q_{\mathrm{o}, k}^{l}-c_{\mathrm{wp}} q_{\mathrm{w}, k}^{l}\right)-\sum_{k=1}^{N_{I}} c_{\mathrm{wi}} q_{\mathrm{wi}, k}^{l}\right] \frac{\Delta t^{l}}{\left(1+r_{d}\right)^{\left(t_{l} / 365\right)}} \\
-\sum_{w=1}^{N_{P}+N_{I}} & \frac{C_{\mathrm{well}}}{\left(1+r_{d}\right)^{\left(t_{w} / 365\right)}},
\end{aligned}
$$

where $C_{\text {well }}$ is the cost of drilling and completing a well, $t_{w}$ is the time of delivering well $w$, $\mathbf{m}_{f}$ is the vector of reservoir model which contains properties such as permeability values at grid blocks, $N_{l}$ is the number of simulation time steps, $t_{l}$ is the simulation time (in days), and $r_{d}$ is the annual discount rate. Variables $N_{P}$ and $N_{I}$ denote the number of producers and injectors, respectively, and $p_{\mathrm{o}}, c_{\mathrm{wp}}$ and $c_{\mathrm{wi}}$ indicate the oil price and the cost of handling produced and injected water (all in $\$ / \mathrm{STB}$ ). Variables $q_{\mathrm{o}, k}^{l}$ and $q_{\mathrm{w}, k}^{l}$ denote oil and water

production rates for producer $k$ at simulation time step $l, q_{\mathrm{wi}, k}^{l}$ is the water injection rate of injector $k$ (all in STBD), and $\Delta t^{l}$ is the size (in days) of simulation time step $l$.

The vector of decision parameters, $\mathbf{x}$, specify the number of wells, their locations and controls, well type (producer/injector) and the sequence in which new wells are drilled. The continuous control variables for new and existing wells are treated similarly in the optimization. For existing wells, only control variables after $t_{i}$ are included in the optimization at $t_{i}$. The location variables are discrete (pseudo-continuous) and treated similar to the continuous variables but are rounded for input into the reservoir simulation. The optimization problem in (1) is solved through a parallelized PSO-MADS optimization algorithm (Isebor et al, 2014b). Nonlinear constraint here is the minimum well distance constraint which is handled through the filter method in PSO-MADS. The PSO-MADS algorithm converges when a maximum number of iterations or a minimum stencil length is reached. This algorithm is applied within the optimization with sample validation (OSV) procedure, discussed next. The initial guess for optimization (through OSV) at $t_{i}$ corresponds to the optimal solution at $t_{i-1}$. This is also demonstrated in Fig. 1 .

In CLFD optimization at any $t_{i}$, the NPV for each model in Eq. 3 is computed from time zero to the end of specified project life. This is to ensure that we can compare the value of the project at different steps. Although the NPV is computed from time zero, the simulation runs are only performed for the duration of $\left[t_{i}, T\right]$ (as the earlier state variables and decision parameters correspond to the past and do not change). For restarting the simulations at $t^{i}$, the state variables for each realization are saved into restart files from the final simulation performed at the model calibration step. We let $\mathbf{x}^{i}$ denote the optimal solution obtained from the optimization at step $i$. Note that other economic measures such as rate of return could be incorporated in the objective function here (Shirangi et al, 2017).

\subsection{Optimization with sample validation and selection of representative models}

We now discuss the optimization with sample validation (OSV) procedure from Shirangi and Durlofsky (2015). Ideally, the optimization should be performed over the full set of 
$N_{R}$ models (here, we set $N_{R}=50$ ). Since the computational cost scales linearly with the number of realizations used, an optimization over the full set will be computationally expensive. Therefore, we perform the optimization over a set of $n_{r}<<N_{R}$ representative models (e.g., $n_{r}=5$ ). We will discuss later how these $n_{r}$ models are selected.

The optimization over the representative subset, improves the initial objective value of $\bar{J}\left(\mathbf{x}_{\text {init }}, M_{\text {rep }}^{i}\right)$ to an optimal value of $\bar{J}\left(\mathbf{x}_{\text {opt }}, M_{\text {rep }}^{i}\right)$. We then apply this optimal solution to the full set of models. The NPV improvement for the full set of models is therefore $\bar{J}\left(\mathbf{x}_{\mathrm{opt}}, M^{i}\right)-\bar{J}\left(\mathbf{x}_{\text {init }}, M^{i}\right)$. The relative improvement, $R I$, is computed as the ratio of NPV improvement for the full set, divided by that computed for the representative subset, i.e.,

$$
R I=\frac{\bar{J}\left(\mathbf{x}_{\mathrm{opt}}, M^{i}\right)-\bar{J}\left(\mathbf{x}_{\text {init }}, M^{i}\right)}{\bar{J}\left(\mathbf{x}_{\mathrm{opt}}, M_{\mathrm{rep}}^{i}\right)-\bar{J}\left(\mathbf{x}_{\text {init }}, M_{\mathrm{rep}}^{i}\right)}
$$

We require $R I \geq \alpha$ to accept $\mathbf{x}_{\mathrm{opt}}$ as the optimal solution at step $i(0 \leq \alpha \leq 1)$ and we set $\alpha=0.5$. If this criterion is not satisfied, we increase $n_{r}$ and repeat the optimization, until $R I \geq 0.5$ is satisfied, or the maximum number of OSV iterations (specified to 4 here) is reached. In this work, a sequence of $\{5,9,16,25\}$ models is considered in OSV, i.e., a PSO-MADS optimization is first performed over $n_{r}=5$ models with $\mathbf{x}_{\mathrm{opt}}$ as the optimal solution; if the $R I$ criterion is not satisfied, the optimization is repeated for a (new) set of $n_{r}=9$ models selected based on the current solution. The OSV procedure is outlined as follows. Note that each OSV iteration, $1 \leq k \leq 4$, involves an order of 100 PSO-MADS iterations at Step 3.

1. At CLFD step $i$, initialize the OSV iteration index to $k=0$, and set $\mathbf{x}_{k}=\mathbf{x}_{\text {init }}=\mathbf{x}^{i-1}$ where $\mathbf{x}^{i-1}$ is the optimal solution from the previous CLFD optimization step. Also set the initial value of $n_{r}$.

2. Select $n_{r}$ representative models from the full set of $N_{R}$ realizations in $M^{i}$ by use of flow simulation results based on $\mathbf{x}_{k}$. Note that for $k>1$, these simulation results are already available from the simulations performed to compute the RI criterion at OSV iteration $k-1$.

3. Solve the optimization problem in (1), using PSO-MADS, with $\mathbf{x}_{k}$ as the initial guess and the optimal solution denoted by $\mathbf{x}_{k+1}\left(=\mathbf{x}_{\mathrm{opt}}\right)$.

4. Compute $R I$ in Eq. 4 by replacing $\mathbf{x}_{\mathrm{opt}}$ with $\mathbf{x}_{k+1}$ and $\mathbf{x}_{\text {init }}$ with $\mathbf{x}^{i-1}$.

5. If $R I \geq 0.5$, accept the optimal solution and set $\mathbf{x}^{i}=\mathbf{x}_{k+1}$.

If the maximum OSV iterations has reached, set $\mathbf{x}^{i}=\arg \max _{\mathbf{x}_{k}} \bar{J}\left(\mathbf{x}_{k}, M^{i}\right)$. Otherwise, set $k=k+1$, increase $n_{r}$, and go to 2 (note that new representative models will be selected based on the new $\mathbf{x}_{\mathrm{opt}}$ ).

The sample validation in OSV is to ensure that the optimal solution obtained for a small representative subset of models adequately improves the objective function for the full set. Other criteria such as out-of-sample validation could also be applied and tested here. For computational results in this work, a sample size of $n_{r}=9$ or 16 typically satisfied $R I \geq 0.5$, while a smaller size was not usually adequate. 
We now discuss the selection of representative models in CLFD. There are various methods presented for realization selection. Here we use a CDF approach as explained in Shirangi (2017). In this approach, flow simulation is performed for the full set of $N_{R}$ models by use of current $\mathbf{x}_{k}$, and the NPV values are computed. The cumulative distribution function $(\mathrm{CDF})$ plot is then generated. The models are selected such that two of them correspond to P10 and P90 (for $n_{r} \leq 10$ ) or P5 and P95 (for $n_{r}>10$ ), and the rest of $n_{r}-2$ models correspond to even increments in NPV percentile (e.g., for $n_{r}=5$, the models correspond to P10, P30, P50, P70, and P90). Other selection methods such as equally weighted flow-based and permeability-based clustering (Shirangi and Durlofsky, 2016 ) could be applied and tested here 1 .

\subsection{CLFD model calibration for channelized models}

In CLFD model calibration step, the $N_{R}$ realizations are updated with new hard data and production data. Conditioning to production data is an inverse problem where one or multiple models need to be generated such that they reproduce the observed flow data and honor prior geological information. In the context of inverse problem theory (Tarantola, 2005), generating multiple calibrated (history-matched) models correspond to a sampling of the posterior probability density function (pdf). A common approach for solving this problem is the randomized maximum likelihood (RML) (Kitanidis, 1995; Shirangi, 2014). With RML, a sample from the posterior pdf is generated by minimizing an objective function that quantifies the mismatch between observed and predicted data. This objective function also has a model mismatch term to preserve the prior geological information. Model calibration can also be accomplished by use of a Kalman filter (Ghorbanidehno et al, 2015 , Ghorbanidehno, 2018).

For model calibration of a channelized reservoir described by MPS, the optimizationbased principal component analysis (O-PCA) parameterization (Vo and Durlofsky, 2015 Liu, 2017) is applied here. The CLFD model calibration for MPS models consists of two steps. In the first step, new conditional realizations are generated using a geostatistical simulation approach with hard data from all wells (including the most recent well). Here we use the multi-scale cross-correlation simulation (MS-CCSIM) geostatistical algorithm (Tahmasebi et al, 2014) for generating these conditional realizations. In the second step, the O-PCA-based RML is applied for conditioning $N_{R}$ realizations to production data. The schematic of model calibration procedure is presented in Fig. 2.

We now briefly describe these procedures. The O-PCA method requires generating $L$ realizations of the permeability field conditioned to hard data at well locations. We use $L=1000$ in this work. New realizations must be generated at each CLFD step since new conditioning data become available as we proceed in time. The MS-CCSIM algorithm is very efficient and can generate these models in a few minutes. Then the centered matrix of realizations, $\mathbf{X}_{\mathrm{c}}$, is computed,

$$
\mathbf{X}_{\mathrm{c}}=\left[\mathbf{m}_{1}-\overline{\mathbf{m}} \ldots \mathbf{m}_{L}-\overline{\mathbf{m}}\right]
$$

where $\overline{\mathbf{m}}$ is the mean of the $L$ realizations. A truncated SVD of $\mathbf{X}_{\mathrm{c}}$ is then computed as

\footnotetext{
${ }^{1}$ the $\mathrm{R}$ code is available here:
} https://github.com/mehrdad-shirangi/representative_model_selection 


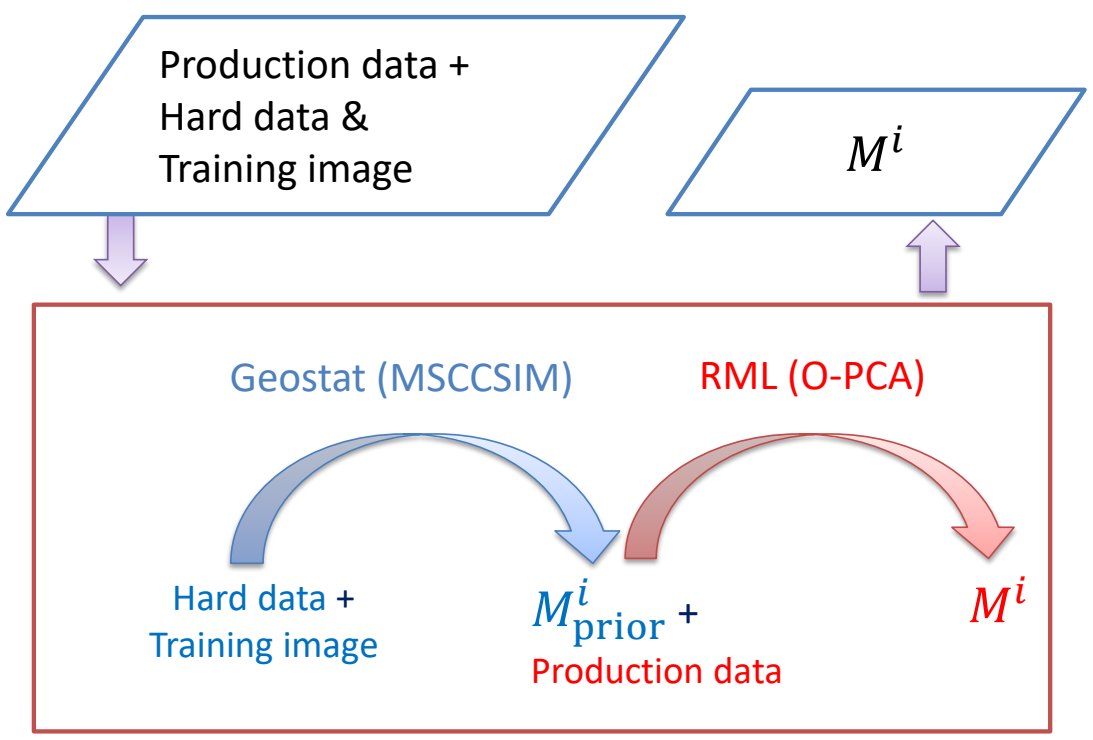

Figure 2: Schematic of CLFD model calibration step with multipoint geostatistics.

$\mathbf{U}_{l} \boldsymbol{\Lambda}_{l} \mathbf{V}_{l}^{T}$, where $l<L$. Given a random $l$-dimensional vector $\boldsymbol{\xi} \sim \mathcal{N}(\mathbf{0}, 1)$, a new realization can be generated by solving the following optimization problem:

$$
\mathbf{m}=\operatorname{argmin}_{\mathbf{z}}\left\{\left\|\mathbf{U}_{l} \boldsymbol{\Lambda}_{l} \boldsymbol{\xi}+\overline{\mathbf{m}}-\mathbf{z}\right\|_{2}^{2}+\gamma R\right\},
$$

where $R$ is a regularization term that is specified such that the realization is generated consistent with the training image (see Vo and Durlofsky (2014) for details). For binary models here, $R=\mathbf{z}^{T}(\mathbf{1}-\mathbf{z})$, where $\mathbf{1}$ is the unity vector of same length as $\mathbf{z}$. The SNOPT algorithm (Gill et al, 2005) is applied to solve (6).

In the O-PCA RML, a calibrated model is generated by minimizing the following objective function.

$$
S(\boldsymbol{\xi})=\frac{1}{2}\left(\boldsymbol{\xi}-\boldsymbol{\xi}_{\mathrm{uc}}\right)^{T}\left(\boldsymbol{\xi}-\boldsymbol{\xi}_{\mathrm{uc}}\right)+\frac{1}{2}\left(\mathbf{g}^{p}(\boldsymbol{\xi})-\mathbf{d}_{\mathrm{uc}}^{p}\right)^{T} C_{d, p}^{-1}\left(\mathbf{g}^{p}(\boldsymbol{\xi})-\mathbf{d}_{\mathrm{uc}}^{p}\right),
$$

where $\boldsymbol{\xi}_{\mathrm{uc}}$ corresponds to a projected MPS realization, i.e., $\boldsymbol{\xi}_{\mathrm{uc}}=\boldsymbol{\Lambda}_{l}^{-1} \mathbf{U}_{l}\left(\mathbf{m}_{\mathrm{uc}}-\overline{\mathbf{m}}\right)$. Here $\mathbf{m}_{\mathrm{uc}}$ is a realization that is unconditioned to production data, but conditioned to hard data $\left(\mathbf{m}_{\text {uc }}\right.$ is also generated by MS-CCSIM). Note that the hard data mismatch term does not appear since hard data are already honored in the realizations and thus in the O-PCA representation. Minimization of Eq. 7 is also performed by SNOPT (Gill et al, 2005). This minimization is to find the optimal $l$-dimensional $\boldsymbol{\xi}$. In the computational results of this work, we will set $l=100$.

Note that for each trial $\boldsymbol{\xi}$, the vector $\mathbf{m}$ is obtained from solving (6). Predicted data, $\mathbf{g}^{p}$, is then generated by performing a reservoir simulation run using this $\mathbf{m}$. The mismatch objective function in Eq. 7 is then computed. The gradient of $S$ with respect to $\mathbf{m}$ is constructed through an adjoint solution, using the automatic differentiation framework (Bukshtynov et al, 2015). The gradient is then projected using the chain rule to obtain derivatives with respect to $\boldsymbol{\xi}$. 


\subsection{Parallelized implementation}

The CLFD algorithm can be parallelized in both the optimization and model calibration steps. In the PSO-MADS algorithm, the objective function for $p$ PSO particles and the $s$ stencil points in MADS algorithm, can be computed simultaneously, by accessing $n_{\text {node }}$ compute nodes. The number of stencil points is twice the number of decision parameters, and $p$ is typically selected to be a comparable number (e.g., $p=s / 3$ or $p=s / 2$ ). See Isebor et al (2014a) for more details on parallelization of PSO-MADS.

In the model calibration step, as the RML runs are independent of one another, each RML realization is generated on a separate compute node using distributed computing. All flow simulations are performed using Stanford's Automatic-Differentiation-based General Purpose Research Simulator (AD-GPRS) (Younis, 2011). The existing OpenMP-based parallelized version of AD-GPRS (Zhou and Tchelepi, 2012) allows us to run each simulation on a computational node with 8 cores. This gives an average speedup of about a factor of 5 for each simulation. For CLFD results presented in this work, at each model calibration step, $N_{R}=50$ posterior RML models are generated simultaneously using 50 compute nodes, providing a speedup factor of 250 compared with a sequential application on a single CPU.

\subsection{TruMAP: True-Model-based Assessment of Performance}

As discussed in Introduction, recent work that applied modified versions of CLFD (Morosov and Schiozer, 2016; Hanea et al, 2017), reported that application of CLFD, in some cases, has resulted in slight reduction of true-model NPV. It may seem counter-intuitive that assimilating new data and re-optimizing decision variables over the remaining span of project life, in cases has lead to a decrease in true-model NPV (which is the ultimate outcome).

It is known in "decision analysis" that a decision is distinct from the outcome and that a good decision does not necessarily lead to a good outcome (Howard, 1988; Bickel and Bratvold, 2008). In fact, the outcome of a closed-loop optimization procedure can be treated as a random variable. Therefore, there is a distribution of feasible outcomes at each optimization step. A sampling of any of these distributions can be obtained by repeating the closed-loop optimization procedure for multiple cases of true-model. The author refers to this procedure as TruMAP which stands for "True-Model-based Assessment of Performance". The TruMAP procedure is outlined as follows.

1. Specify $n_{\mathrm{m}}$, the number of true-model cases, and select $n_{\mathrm{m}}$ candidate models, randomly, from the prior set.

2. For each of the $n_{\mathrm{m}}$ true-model cases, perform the closed-loop optimization (CLFD, here) after an optimization over the prior models.

3. Compute all performance measures for each of $n_{\mathrm{m}}$ cases.

4. Present the distribution obtained from these $n_{\mathrm{m}}$ runs for each performance measure. Following the arguments in Simmons et al (2011), it is essential not to cherry-pick the results, or eliminate any runs, or reduce $n_{\mathrm{m}}$ here. 
We will define performance measures for CLFD and apply the above procedure in the computational results later in this paper. Note that it is important to perform the optimization over the prior models for each true-model case as noted in Step 2 of the above procedure.

The use of multiple true-models in CLFD has also been considered by Hanea et al (2017) and Shirangi and Durlofsky (2015). Hanea et al (2017), however, did not present a procedure for performance assessment (as TruMAP) and used too few cases. Barros et al. (2016) also applied closed-loop optimization for multiple true-model cases, though in a different context, i.e., quantifying value of information in CLRM.

The author believes that the TruMAP procedure can help eliminate the unfortunate tendency to assess an algorithm performance for a few true-model cases and only report the results supporting the significance of the algorithm/hypothesis. The interested reader is encouraged to refer to Simmons et al (2011) for an excellent discussion and a suggested solution on this.

\section{Computational results and discussion}

In this section computational results are presented for a binary channelized system. In Part 1, detailed application of CLFD is presented. In Part 2, multiple CLFD runs are performed for performance assessment through TruMAP.

\subsection{Part 1: CLFD for a channelized model}

This example involves a reservoir model described on a two-dimensional uniform grid of dimensions $60 \times 60$ with $\Delta x=\Delta y=100 \mathrm{ft}, \Delta z=15 \mathrm{ft}$. A binary channelized training image from Vo and Durlofsky (2014) is taken as the prior geological description (Fig. 3), from which a set of unconditional realizations are generated using the MS-CCSIM geostatistical algorithm (Tahmasebi et al, 2014). The realizations are not constrained to honor the sand/shale ratio observed in the training image. The sand permeability is $500 \mathrm{mD}$, while the shale permeability is $10 \mathrm{mD}$. The true permeability field, along with an initial guess for the well locations $\left(\mathbf{x}^{0}\right)$, is shown in Fig. 4. Three prior realizations of the permeability field are shown in Fig. 5. Porosity is assumed to be constant and equal to 0.2. Initially, the reservoir is at irreducible water saturation which is equal to $S_{w c}=0.2$.

Project life is 3000 days, which is divided into seven control steps, with the first five control steps of length 180 days, and the last two control steps of length 1050 days. In this example, we assume that two rigs are available $\left(n_{\text {rig }}=2\right)$ and therefore (a maximum of) two wells can be drilled at each CLFD step. The optimal number of wells, however, is determined from the optimization. The drilling time is specified as $T_{\text {drill }}=180$ days. Therefore, the $t_{i}$ values are given by $\{0,180,360,540,720\}$. The last optimization is performed at 540 days, which determines the decision parameters corresponding to well type and location of wells $9 \& 10$ and (future) BHPs of all wells (wells 1-8). Discount rate is specified to $r_{d}=0$. Simulation and optimization parameters are presented in Table 1, and relative permeability curves are shown in Fig. 6. The simulation input files, permeability realizations, and the training image are available online at https://purl.stanford.edu/nn001cr1881. 


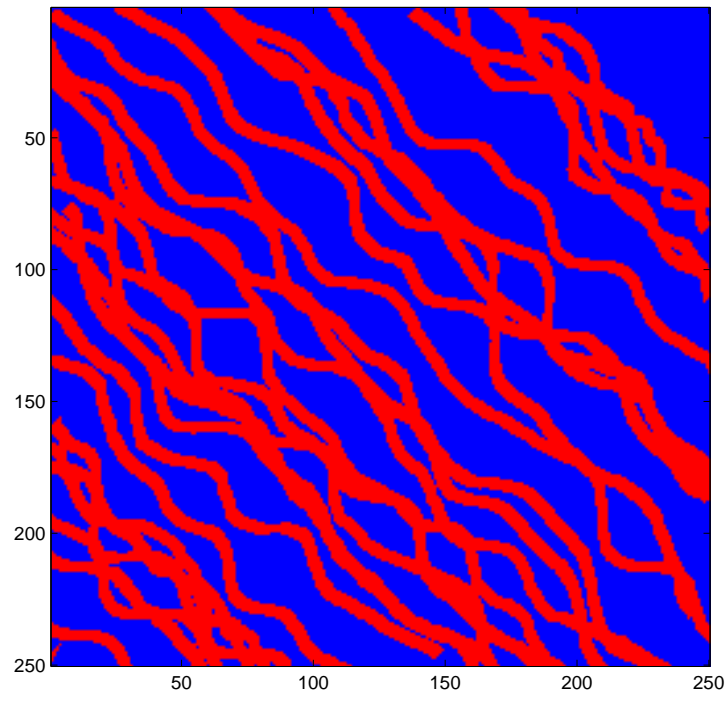

Figure 3: Training image used to generate all permeability models (together with any available hard data).

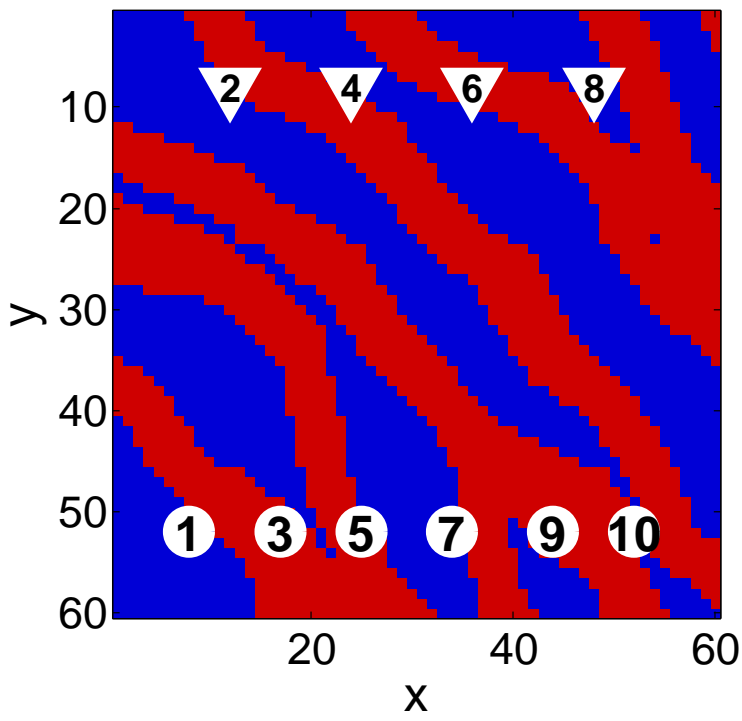

Figure 4: True permeability field, with red indicating sand facies (permeability of $500 \mathrm{mD}$ ), and blue indicating shale facies (permeability of $10 \mathrm{mD}$ ). The initial well configuration is also shown with circles denoting producers and triangles denoting injectors (Part 1). 


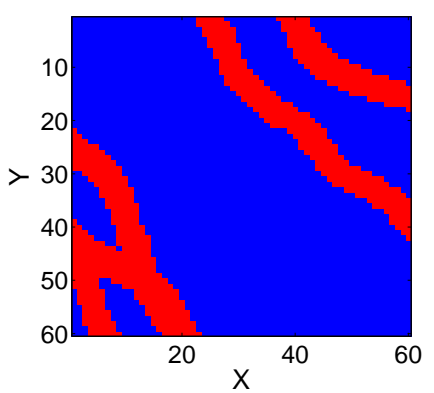

(a) Realization 1

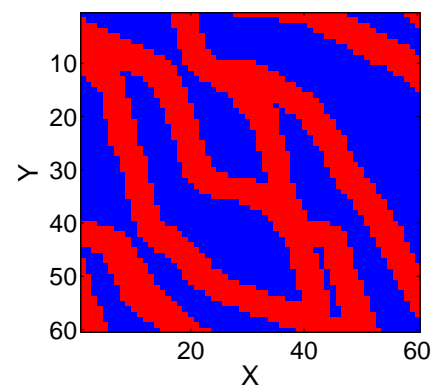

(b) Realization 2

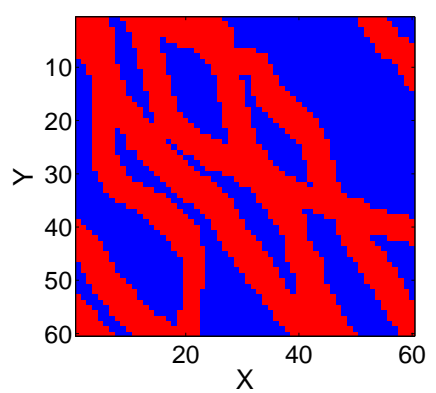

(c) Realization 3

Figure 5: Three prior realizations of permeability field, with red indicating sand facies (permeability of $500 \mathrm{mD}$ ), and blue indicating shale facies (permeability of $10 \mathrm{mD}$ ).

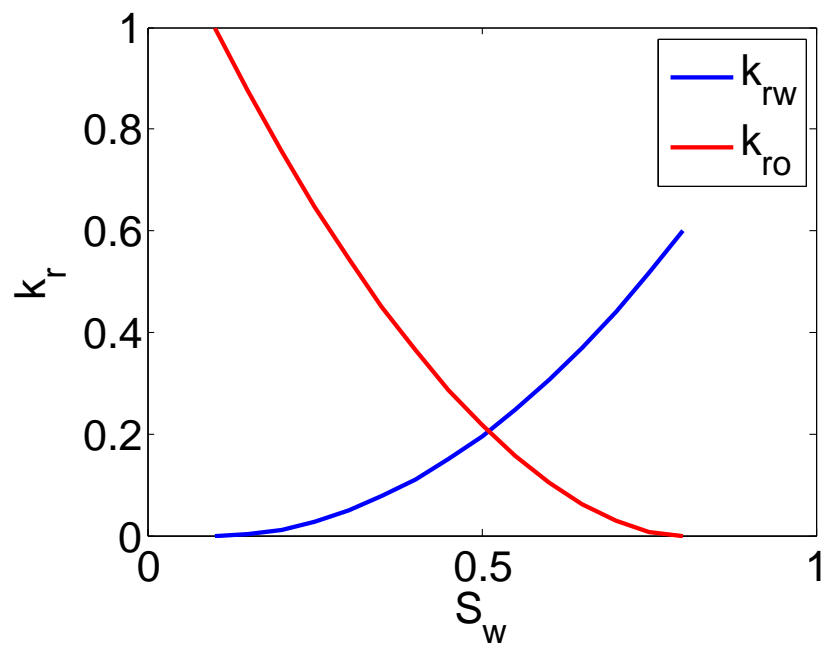

Figure 6: Oil and water relative permeability curves.

Table 1: Optimization parameters for all examples

\begin{tabular}{lc}
\hline Parameter & Value \\
\hline$C_{\text {well }}$ & $\$ 10 \mathrm{MM}$ \\
$r_{o}$ & $\$ 90 \mathrm{STB}$ \\
$c_{w p}$ & $\$ 10 \mathrm{STB}$ \\
$c_{w i}$ & $\$ 10 \mathrm{STB}$ \\
Prod BHP range & $1000-4100 \mathrm{psi}$ \\
Inj BHP range & $4600-7000 \mathrm{psi}$ \\
\hline
\end{tabular}




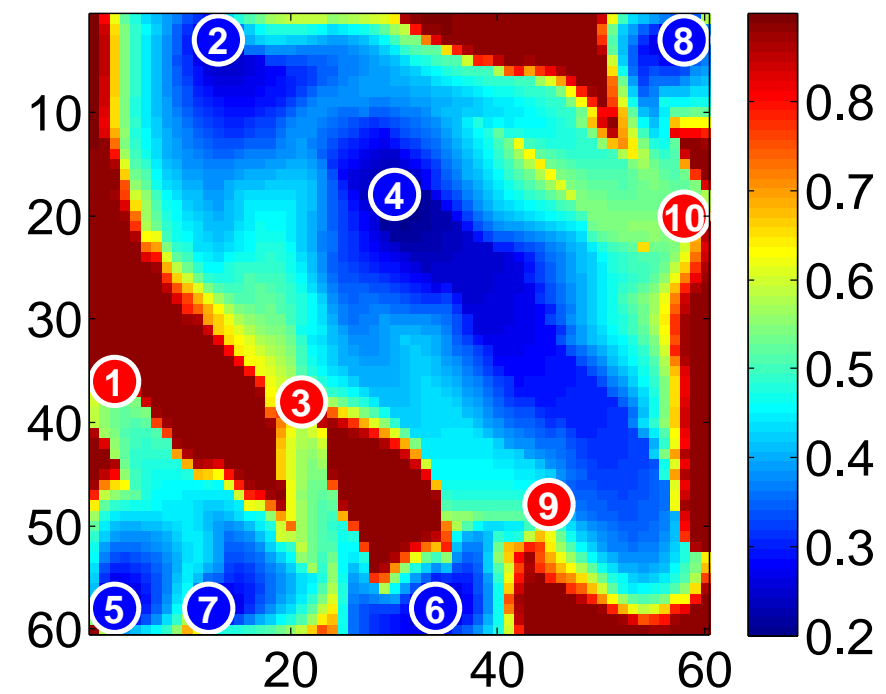

Figure 7: Well configuration from deterministic optimization (using $\mathbf{m}_{\text {true }}$ ), with red denoting producer, blue denoting injector, and the well numbers indicating the drilling sequence. Background shows final oil saturation. Note that two wells are drilled at a time.

The number of decision parameters is 80 . This corresponds to 10 categorical variables for well types, 20 integer variables for well locations, and 50 continuous parameters for well settings/controls (BHP). Not all wells exist at all control steps. Specifically, in the first control step, only two wells appear in the model. Similarly, four and six wells exist in the second and third control steps, respectively. Therefore, the total number of continuous control parameters is $2+4+6+8+10+10+10$ or 50 . The initial guess for control parameters correspond to the average of upper bounds and lower bounds. Well type can take values in $\{-1,0,1\}$ which corresponds to \{producer, do not drill, injector $\}$ (for more details please refer to Isebor et al (2014b)). In each optimization, the PSO-MADS algorithm is applied with 60 PSO particles, and the minimum mesh size for MADS is specified to be $1 \%$ of the variable range. In the evaluation of a trial point during optimization, if the well distance constraint is violated, simulation run is not performed and a zero objective function value is assigned to that point. This treatment is to avoid frequent failed simulation jobs that happens for trial points with two wells located too close or at the same grid blocks. In addition, economic constraint for each producer is enforced by the simulator by specifying a maximum WOR (water to oil ratio).

First, deterministic optimization is applied using the true-model (shown in Fig 4). The optimal development plan and the final oil saturation map are shown in Fig. 7. The NPV from the initial guess is $\$ 441 \mathrm{MM}$ while the optimal NPV is $\$ 783 \mathrm{MM}$. Next, CLFD using OSV is applied for this case.

CLFD is applied following the workflow shown in Fig. 1. Observed data for CLFD model calibration include production data (oil and water production rates at producers and water injection rate at injectors) measured at 30-day intervals and hard data from all existing wells. Observed production data is generated by adding random noise (measurement error) 


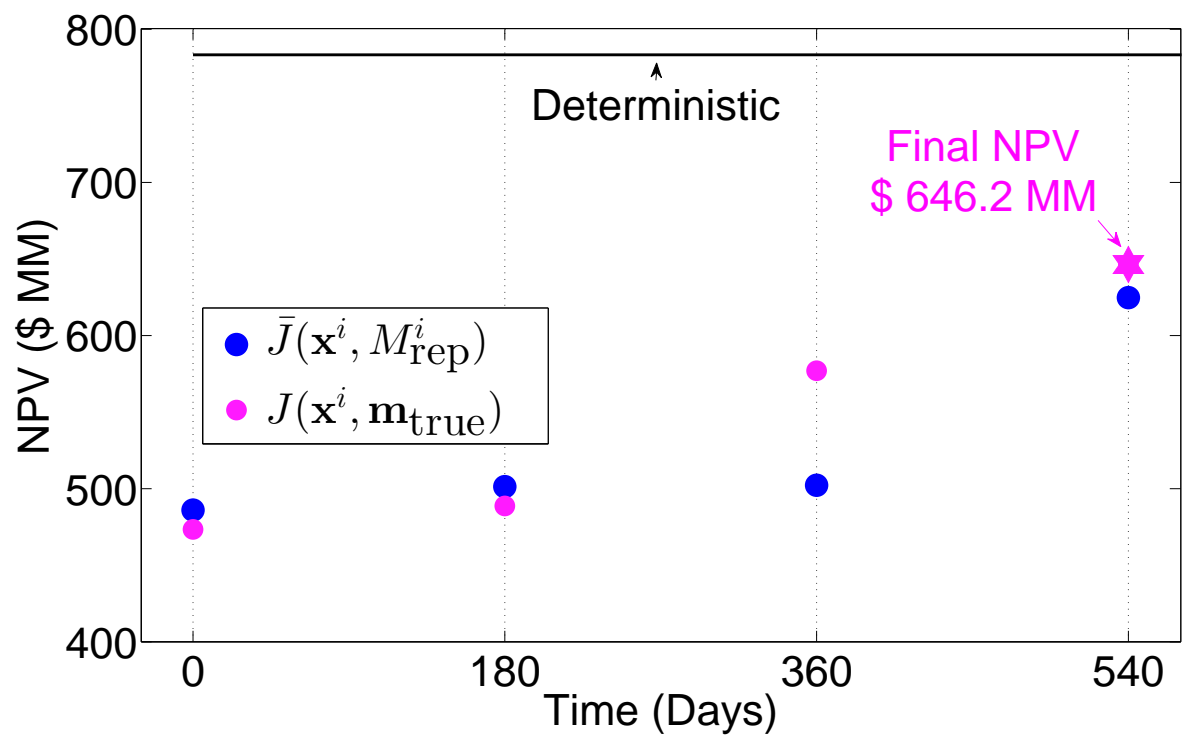

Figure 8: Optimal expected NPV, and the corresponding NPV for the true-model, versus CLFD step. The number of realizations at each CLFD step is determined using OSV. The star shows the final true NPV from CLFD.

to the true data, where the true data corresponds to flow results of the true model when it is simulated with the optimal solution. Model calibration is performed every 180 days by first generating new geological realizations conditioned to hard data using the MS-CCSIM algorithm, and then applying O-PCA-based RML using all production data from time 0 .

The progress of the true NPV with CLFD step is shown in Fig. 8. In the evaluation of the true NPV, a nominal strategy is applied where a producer is shut in when the cost of handling produced water exceeds the revenue from oil. The NPV from the deterministic optimization is also shown here for comparison. The final truth-case NPV from CLFD is $\$ 646.2 \mathrm{MM}$, which is $36.6 \%$ higher than the NPV from optimization (using OSV) over prior realizations.

Fig. 9 presents the P10-P50-P90 results for NPV, determined by simulating all 50 realizations and then constructing the cdf, at each CLFD step. The expected NPV based on the current representative subset (which satisfies validation criterion of $R I \geq 0.5$ ) is also displayed. It is evident that the optimal expected NPV for the representative subset falls within the P10-P90 range. Note that in Fig. 9, the percentile values are determined for the updated models $\left(M^{i}\right)$ based on current $\mathbf{x}^{i}$ at each CLFD step $i$. Therefore, the uncertainty range may not necessarily shrink as both the models and the solution are evolving.

Fig. 10 shows the evolution of the well configuration and the geological model for two realizations. The well scenario involves three injectors at $t_{1}$, but four injectors at later times. Each realization continues to show differences though the CLFD steps due to conditioning to new hard and production data.

It may be worth comparing the final oil saturation from final CLFD solution and from the solution obtained by optimization over prior models through OSV (Fig. 11). It is evident that the solution from CLFD corresponds to improved sweep of oil in this case. 


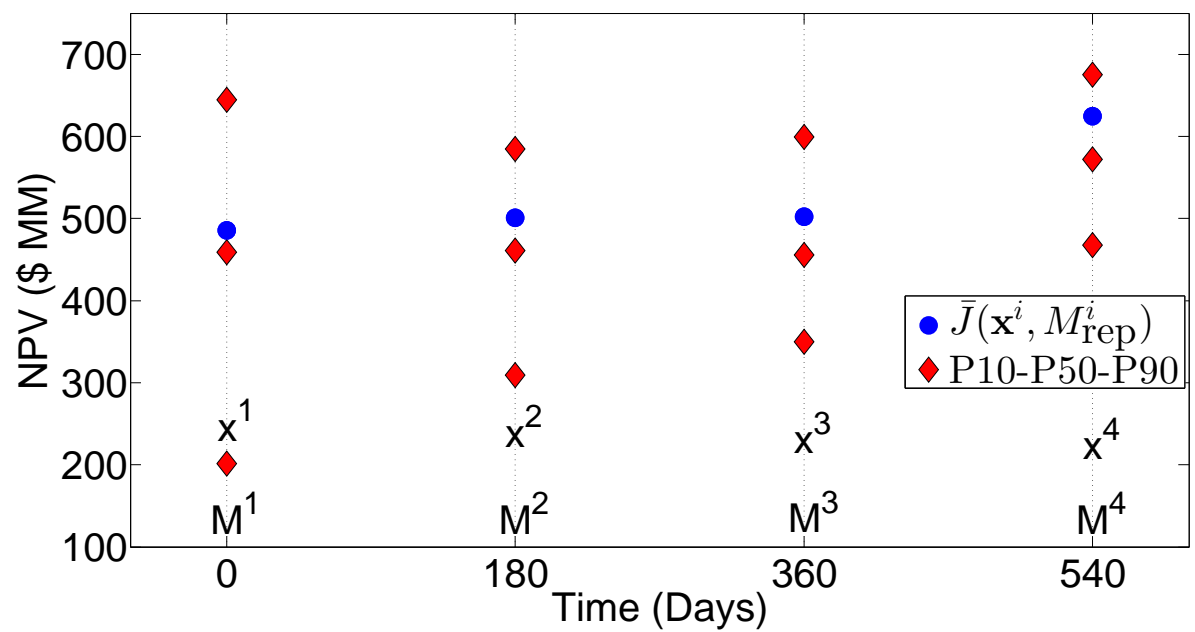

Figure 9: P10, P50, P90 NPVs evaluated for the entire set of 50 realizations, along with the expected NPV for the representative subset, versus CLFD step.

Further, the CLFD solution also corresponds to both higher oil production and NPV as shown in Fig. 12. It appears that the optimal solution from OSV only slightly improves the final NPV compared to the initial guess, whereas the CLFD solution displays a significant improvement.

As discussed earlier, the CLFD model calibration step involves integrating production data from all previous wells (except the most recent wells) together with hard data from all wells including the most recent wells. Integrating both hard data and production data is required to achieve optimal CLFD performance. At each CLFD model calibration step, new realizations conditioned to hard data are generated. We let $\overline{\mathbf{m}}_{\text {prior }}$ designate the mean of these $N_{R}=50$ realizations (conditioned to all available hard data) at each update step. Figs. 13(a) 13(d) show the evolution of the prior mean with CLFD step. We also compute the mean of $N_{R}=50$ posterior realizations (conditioned to both production and hard data). These are shown in Figs. 13(e) 13(h), The optimal development plan at each CLFD step is also shown. It is evident that the mean of the posterior realizations (conditioned to production data) more closely resembles the true-model (Fig. 4). This indicates the importance of integrating production data to reduce the uncertainty in the geological description.

In terms of computational cost, this CLFD application involved a total number of 356,500 simulation runs. As computations were performed on a cluster with access to maximum 400 compute cores, the parallelized computations is equivalent to 1,151 sequential simulation runs (calls to cluster) where each batch of simulations takes about 104 seconds on average. The total computational time is about 32 hours. Note that in practice, each CLFD step is performed at a different time and thus the computational cost will be a fraction of the total cost here. 


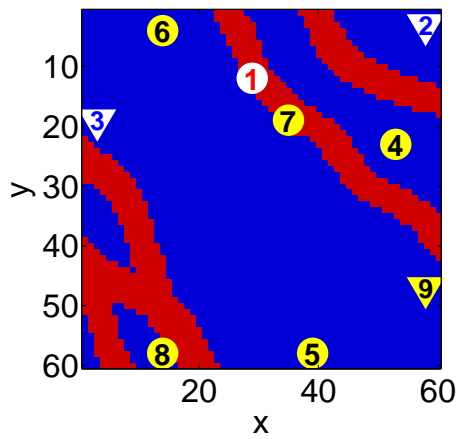

(a) Realization 1 at $t_{0}$ with $\mathbf{x}^{0}$

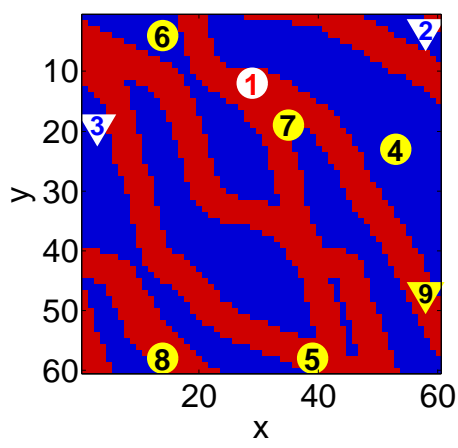

(d) Realization 2 at $t_{0}$ with $\mathbf{x}^{0}$

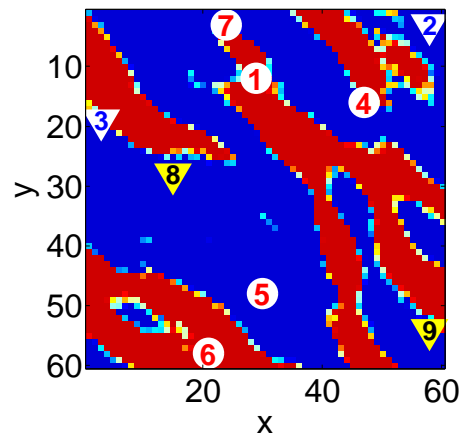

(b) Realization 1 at $t_{2}$ with $\mathbf{x}^{2}$

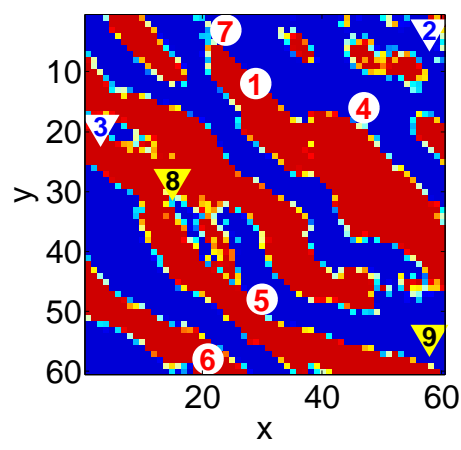

(e) Realization 2 at $t_{2}$ with $\mathbf{x}^{2}$

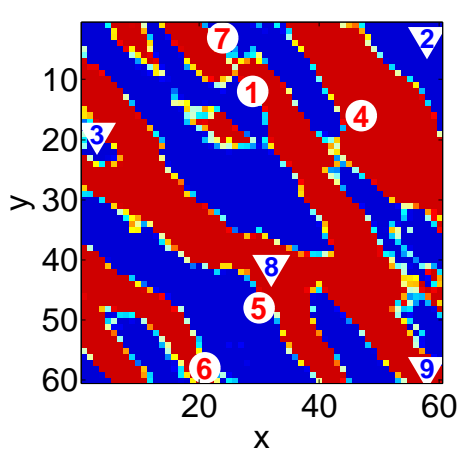

(c) Realization 1 at $t_{3}$ with $\mathbf{x}^{3}$

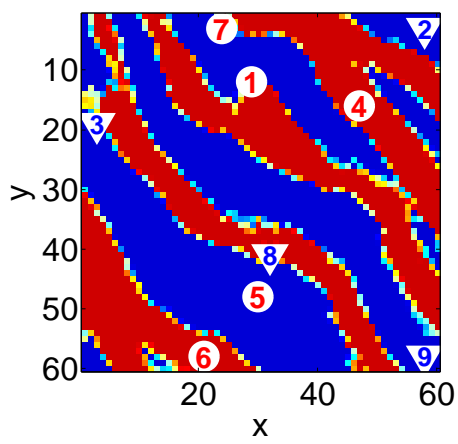

(f) Realization 2 at $t_{3}$ with $\mathbf{x}^{3}$

Figure 10: Evolution of two RML realizations for different CLFD steps, with red indicating sand facies (permeability of $500 \mathrm{mD}$ ), and blue indicating shale facies (permeability of $10 \mathrm{mD}$ ). Current optimal well configuration and drilling sequence is also depicted. Solid white circles and triangles denote producers and injectors (drilled or in the process of being drilled), and yellow circles and triangles denote planned producers and injectors. Numbers indicate the drilling sequence (Example 1). 


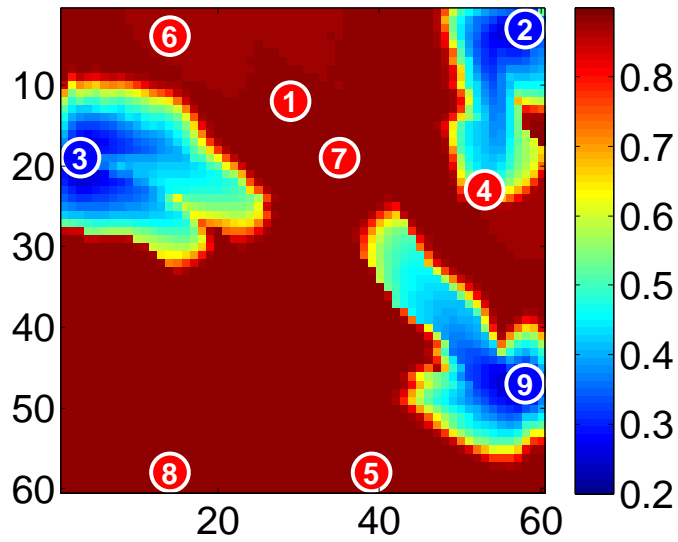

(a)

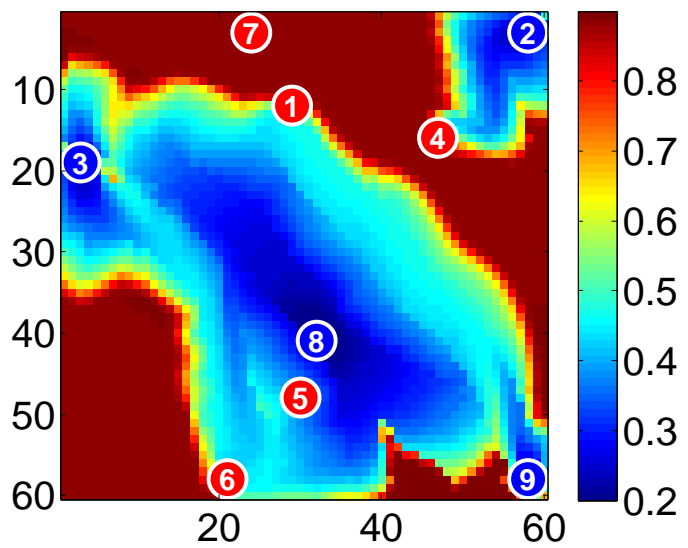

(b)

Figure 11: Final oil saturation from (a) optimization over prior models by OSV, and (b) final CLFD solution. Corresponding well configuration is also shown, with red denoting producer, blue denoting injector, and the well numbers indicating the drilling sequence.

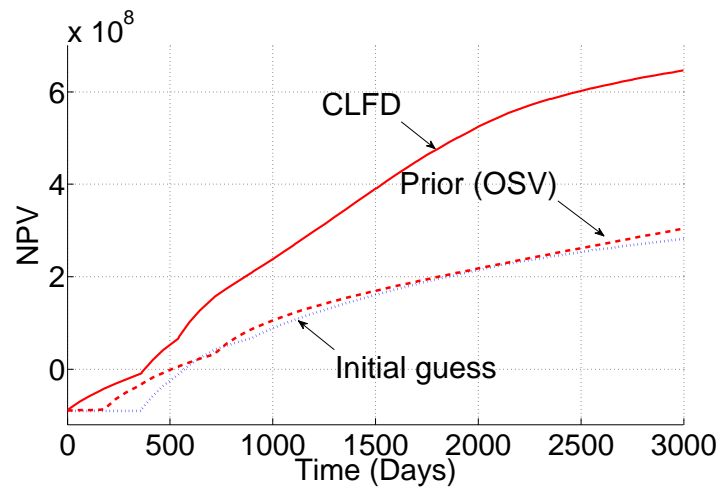

(a)

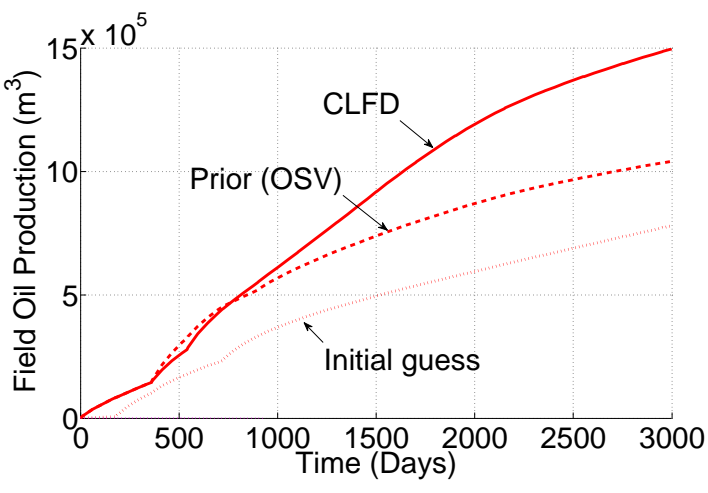

(b)

Figure 12: (a) NPV trajectory, and (b) cumulative oil production, from different approaches. 


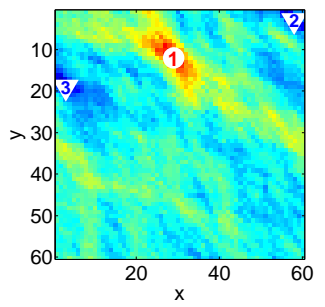

(a) $\overline{\mathbf{m}}_{\text {prior }}, 180$ days

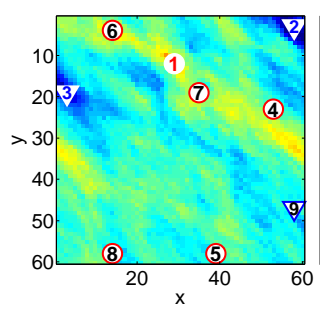

(e) $\overline{\mathbf{m}}_{\text {post }}, 180$ days

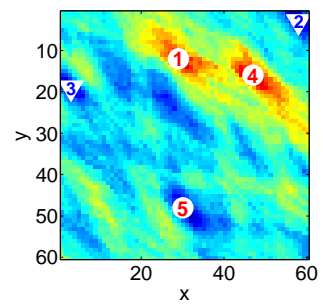

(b) $\overline{\mathbf{m}}_{\text {prior }}, 360$ days

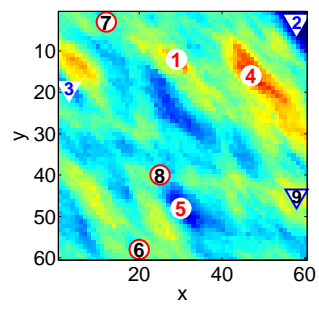

(f) $\overline{\mathbf{m}}_{\text {post }}, 360$ days

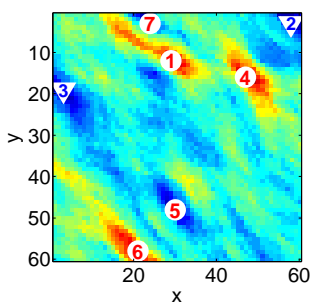

(c) $\overline{\mathbf{m}}_{\text {prior }}, 540$ days

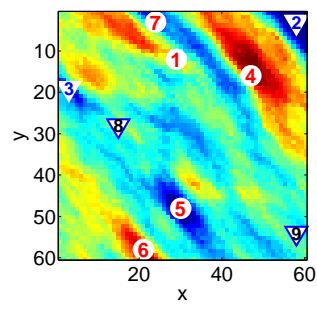

(g) $\overline{\mathbf{m}}_{\text {post }}, 540$ days

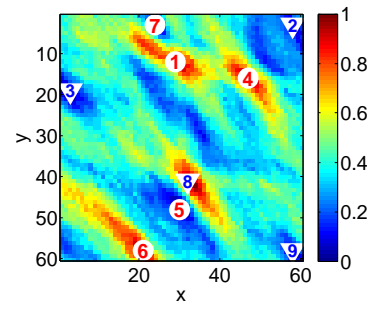

(d) $\overline{\mathbf{m}}_{\text {prior }}, 720$ days

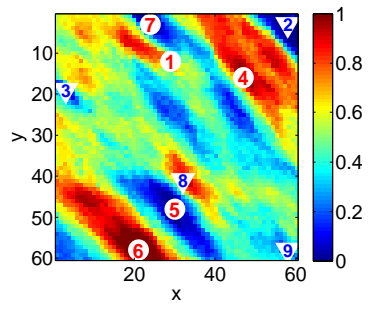

(h) $\overline{\mathbf{m}}_{\text {post }}, 720$ days

Figure 13: Evolution of mean of $\left(N_{R}=50\right)$ prior realizations (conditioned to hard data) and mean of $\left(N_{R}=50\right)$ posterior realizations of facies distribution, for different CLFD steps. Current optimal well configuration and drilling sequence is also depicted. White circles and triangles denote producers and injectors, respectively. Wells with colored (red or blue) numbers are drilled, while outlined red circles and blue triangles denote planned producers and injectors. For the prior model (a-d) only the drilled wells are shown. Numbers indicate the drilling sequence. 
Table 2: NPV ( $\$$ MM) values from optimization over $n_{r}=5$ prior realizations and by use of OSV (where $n_{r}$ is increased to satisfy $R I>0.5$ ) for prior realizations, for five different true models.

\begin{tabular}{|c|c|c|c|}
\hline True model & $J\left(\mathbf{m}_{\text {true }}, \mathbf{x}_{\text {opt }}\right)$ with $n_{r}=5$ & $J\left(\mathbf{m}_{\text {true }}, \mathbf{x}^{i=0}\right)$ from OSV $(\mathrm{RI} \geq 0.5)$ & final $n_{r}$ \\
\hline 1 & 474 & 473 & 9 \\
\hline 6 & 462 & 376 & 16 \\
\hline 11 & 486 & 461 & 9 \\
\hline 16 & 402 & 553 & 9 \\
\hline 21 & 318 & 323 & 9 \\
\hline Avg (1-25) & 339 & 392 & 12.5 \\
\hline Median (1-25) & - & - & 9 \\
\hline
\end{tabular}

\subsection{Part 2: TruMAP for Statistical assessment of CLFD performance}

In this section, our objective is to assess the CLFD and the OSV performance through application with multiple truth-case models. Following the TruMAP procedure, we specify $n_{\mathrm{m}}=25$ and consider 24 additional $\mathbf{m}_{\text {true }}$ models. We repeat the CLFD application for each of these true-models so as to assess the performance in terms of various measures as follows.

\subsubsection{Optimization with sample validation results}

We first discuss the OSV results. We only consider the optimization over prior models $(i=$ 0 ) here. The OSV procedure systematically determines adequate number of representative models in optimization over the full set of realizations (which is 50 here). The results for five of the $n_{\mathrm{m}}=25$ cases are shown in Table 2 (the first row corresponds to the true-model discussed earlier). The average and median $n_{r}$ in this case are 12.5 and 9 , respectively (Table 2). We conclude that for this problem, $n_{r}=5$ is typically not sufficient, whereas optimization over $n_{r}=9$ or 16 realizations is more likely to satisfy the OSV validation criterion. We also observe that the average true-model NPV with OSV is $15.6 \%$ greater than that with $n_{r}=5$ prior models.

\subsubsection{Assessment of single-step CLFD performance}

We now assess the performance of CLFD in terms of improvement of true-model NPV from a single CLFD step. The NPV values for 5 models at each CLFD step are compiled in Table3. The average true NPV from 25 runs (also shown in Table 3 increases monotonically with CLFD step. The average true NPV at CLFD step 1 is $9.9 \%$ greater than optimization over prior $(i=0)$, and the increase from step 1 to 2 and from step 2 to 3 is $7.4 \%$ and $10.1 \%$, respectively. At each CLFD step $i(i \geq 1)$, we define the NPV improvement for true-model $j$ as

$$
d J_{\text {true }, j}^{i}=\frac{J\left(\mathbf{m}_{\text {true }, j}, \mathbf{x}_{j}^{i}\right)-J\left(\mathbf{m}_{\text {true }, j}, \mathbf{x}_{j}^{i-1}\right)}{J\left(\mathbf{m}_{\text {true }, j}, \mathbf{x}_{j}^{i-1}\right)},
$$


Table 3: NPV values $\left(J\left(\mathbf{m}_{\text {true }, j}\right)\right.$ in $\$$ MM) from OSV over prior models and at each CLFD step. Average values are also provided.

\begin{tabular}{lcccc}
\hline True model & OSV-prior $(i=0)$ & $t=180(i=1)$ & $t=360(i=2)$ & $t=540(i=3)$ \\
\hline 1 & 473 & 488 & 577 & 646 \\
6 & 376 & 376 & 314 & 406 \\
11 & 461 & 289 & 449 & 570 \\
16 & 553 & 498 & 529 & 532 \\
21 & 323 & 518 & 596 & 602 \\
Avg $(1-25)$ & 392 & 431 & 463 & 510 \\
\hline
\end{tabular}

Table 4: $\quad$ Statistics of $d J_{\text {true }}^{i}$ (true NPV improvement after one step of CLFD) for 25 different true models

\begin{tabular}{lccccc}
\hline CLFD step (time) & mean & P10 & P50 & P90 & fraction positive \\
\hline$i=1$ (180 days) & 0.14 & -0.18 & 0.11 & 0.55 & 0.64 \\
$i=2$ (360 days) & 0.10 & -0.16 & 0.10 & 0.42 & 0.76 \\
$i=3$ (540 days) & 0.13 & -0.01 & 0.06 & 0.46 & 0.80 \\
\hline
\end{tabular}

where the numerator is the NPV improvement for true-model $j$ as a result of performing one step of CLFD ( $\operatorname{step} i$ ). The mean and percentile values of $d J_{\text {true }, j}^{i}$ are compiled in Table 4 . The mean improvement varies between 10-14\%, and the P10 monotonically increase with CLFD step (Table 4).

The probability of increasing true-model NPV through a single-step of CLFD is simply the fraction of positive $d J_{\text {true }, j}^{i}$ values (last column of Table 4). CLFD improved the truemodel NPV for $64 \%$ of cases at $i=1,76 \%$ of cases at $i=2$, and $80 \%$ of cases at step $i=3$. This performance improvement can also be observed visually in Fig. 14 . Therefore, we observe that although application of a single-step CLFD may even reduce the true NPV of the project in some cases, the chance of increasing true NPV increases at later steps.

At $i=1$ ( $t=180$ days), the uncertainty reduction in model description through integration of spatial data (hard data from four wells) and temporal data (production data from two wells) is not as significant as later steps. As a result, there is $36 \%$ chance of lowering true-model NPV through a single CLFD step. At later steps, however, more wells are drilled and more data results in improved model description. Therefore, there is a higher chance that the optimization over the updated models would result in a higher project outcome (true NPV).

\subsubsection{Assessment of multi-steps CLFD performance}

To further assess the overall net impact of performing multiple steps of CLFD, we define the relative improvement with respect to optimization over prior as

$$
d J_{\text {rel-pr }, j}^{i}=\frac{J\left(\mathbf{m}_{\text {true }, j}, \mathbf{x}_{j}^{i}\right)-J\left(\mathbf{m}_{\text {true }, j}, \mathbf{x}_{j}^{i=0}\right)}{J\left(\mathbf{m}_{\text {true }, j}, \mathbf{x}_{j}^{i=0}\right)},
$$




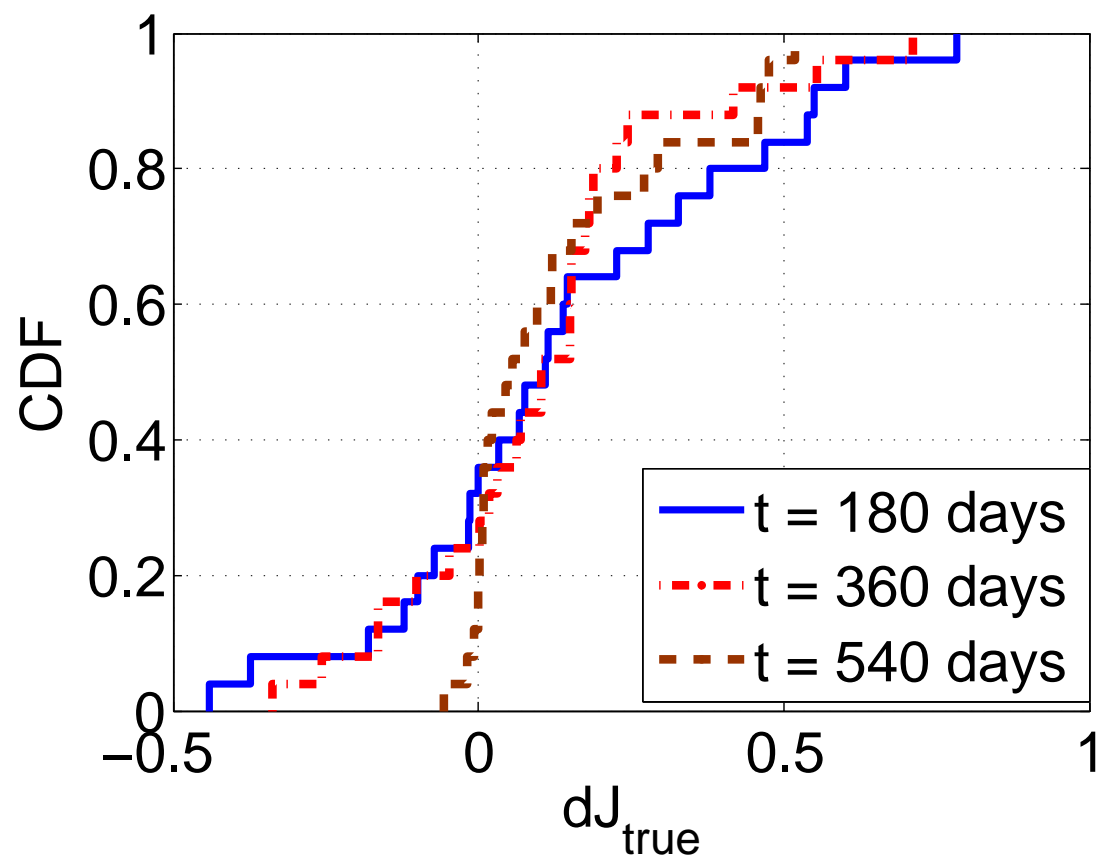

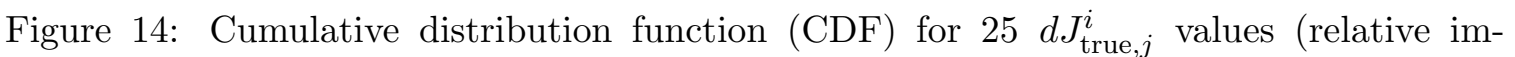
provement in true-model NPV from one-step of CLFD run) for $i=1$ ( $t=180$ days), $i=2$ ( $t=360$ days), and $i=3$ ( $t=540$ days). 


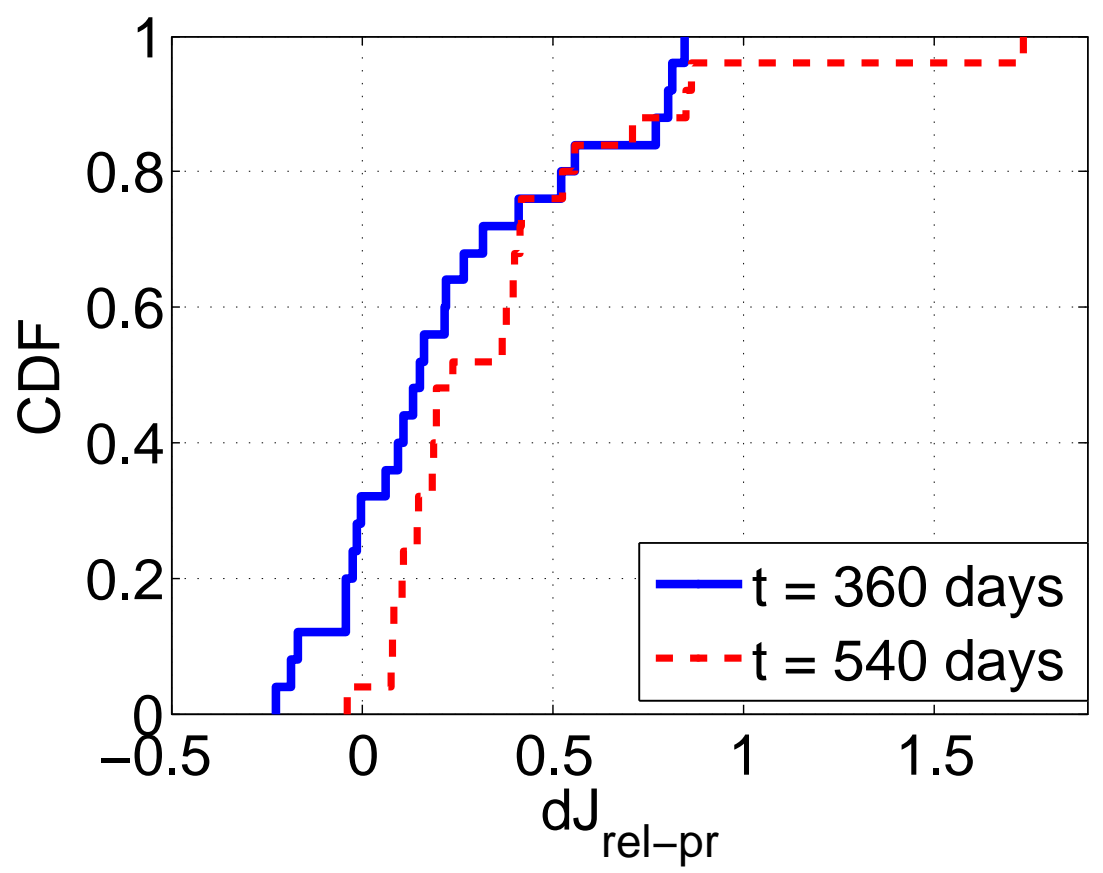

Figure 15: Cumulative distribution function (CDF) for $25 d J_{\text {rel-pr }}^{i}$ values (improvement in true-model NPV relative to optimization over prior models).

where rel-pr stands for "relative to prior". For $i=1, d J$ in Eq. 9 provides the same quantity as that in Eq. 8. However, at later steps $(i=2$ and $i=3)$, these two quantities are different.

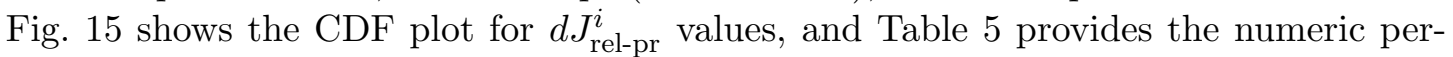
centiles. These results indicate that application of two CLFD steps, has improved the NPV of $68 \%$ of the models, with an average improvement of $23 \%$. The full CLFD run (three CLFD steps in this case), improved the true NPV in $96 \%$ of cases with an average improvement of $37 \%$.

These results together with those in the previous section (and Table 4), indicate that the probability of increasing true model NPV increases with CLFD step. In other words, there is a higher chance of improving true NPV through multi-step CLFD, compared with a single step of CLFD. In this case, we observe that application of one, two, and three consecutive steps of CLFD improved the NPV in $64 \%, 68 \%$, and $96 \%$ of case, respectively. These results infer about an implicit correction mechanism in CLFD that decisions about planned wells (those to be drilled at later steps in the time horizon) and future controls are ultimately improved.

The average percentiles and the average expected NPV, for these 25 runs, versus the CLFD step is shown in Fig 16 and numerical values are provided in Table 6. Each point in the figure (and table) is an average of 25 values (results in Fig. 9 shows values for run $j=1)$, e.g., $<\bar{J}\left(\mathbf{x}^{i}, \mathbf{m}_{\text {true }}\right)>=\frac{1}{25} \sum_{j=1}^{25} J\left(\mathbf{x}_{j}^{i}, \mathbf{m}_{\text {true }, j}\right)$ where $\mathbf{x}_{j}^{i}$ is the optimal solution at CLFD step $i$ for run $j$, and $\mathbf{m}_{\text {true }, j}$ is true-model $j$. These results indicate a monotonic increase in all average values. Further, the difference between the average P10 and the 
Table 5: Statistics of $d J_{\text {rel-pr }}^{i}$ (true-model NPV improvement at each CLFD step $i$ relative to optimization over prior models) for 25 different true models

\begin{tabular}{lccccc}
\hline CLFD step (time) & mean & P10 & P50 & P90 & fraction positive \\
\hline$i=1$ (180 days) & 0.14 & -0.18 & 0.11 & 0.55 & 0.64 \\
$i=2$ (360 days) & 0.23 & -0.17 & 0.15 & 0.80 & 0.68 \\
$i=3$ (540 days) & 0.37 & 0.08 & 0.24 & 0.85 & 0.96 \\
\hline
\end{tabular}

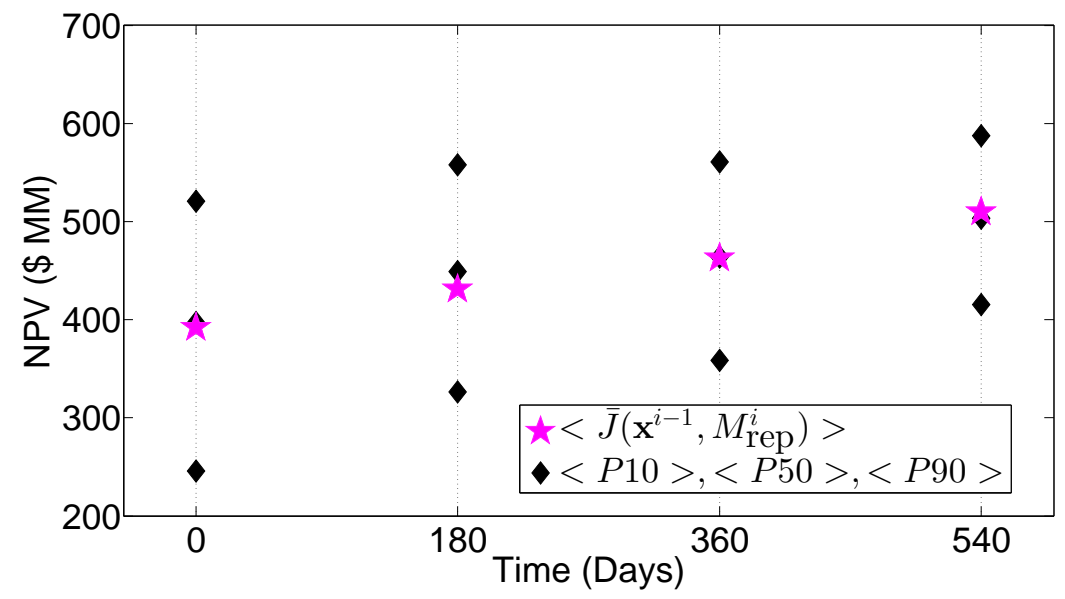

Figure 16: Average values of P10, P50, P90 NPVs, over 25 runs along with the average true NPV, versus CLFD step.

average P90 shrinks with CLFD step, although this does not necessarily hold for each run. These results are not specific to a particular true-model, and therefore they demonstrate the overall performance of CLFD and present an assessment of project value under optimal development.

The author believes that this analysis can help guide investment decisions to lease/buy an asset and in contract decisions between independent oil companies (IOCs) and national oil companies (NOCs). In this example, an investor may lease this reservoir for $\$ 246$ million $\left(<P 10>\right.$ for OSV over prior), knowing that with CLFD, $<P 10>$ at $t^{i=3}$ is $\$ 415$ million.

The computational experiment of performing 25 CLFD applications took about 789 hours

Table 6: Numerical values of average P10, P50, P90 NPVs, over 25 runs along with the average true NPV, versus CLFD step.

\begin{tabular}{lcccc}
\hline & OSV-prior $(i=0)$ & $t=180(i=1)$ & $t=360(i=2)$ & $t=540(i=3)$ \\
\hline$<P 90>$ & 521 & 558 & 561 & 588 \\
$\langle P 50>$ & 397 & 449 & 464 & 504 \\
$\langle P 10>$ & 246 & 326 & 358 & 415 \\
$\left\langle\bar{J}\left(\mathbf{x}^{i}, \mathbf{m}_{\text {true }}\right)>\right.$ & 392 & 431 & 463 & 510 \\
\hline
\end{tabular}


(33 days). The total number of simulations is 9,333,900 which are performed through 30,136 calls to a computer cluster with access to maximum 400 cores.

\subsection{Discussion}

The results for performance assessment showed a high range of possible outcomes from CLFD runs. The highly nonlinear nature of the optimization step is perhaps the main cause of the wide range of variability in the ultimate outcome. Although, the application of PSO component in the PSO-MADS algorithm, provides some degree of global exploration, the optimization may ultimately converge to a locally optimal solution (for the representative subset) and there is no guarantee of finding the global optimum. Results presented in Onwunalu and Durlofsky (2010) show that for a problem where the location of a single well is optimized, the objective function surface is highly rough with many local optima. The field development optimization problem in CLFD is significantly more complex, and a significant number of local optima may exist. Another main cause to the wide range of final outcome, is the sparsity in spatial data and the inherent uncertainty in model description after model calibration. Despite all the complexity, application of the full CLFD improved the true NPV in $96 \%$ of cases, proving the effectiveness of the framework.

The statistical performance assessment procedure can be applied to assess the value of a subsurface reservoir under optimal CLFD. Decision making depends on the risk attitude and therefore, presenting a range of possible outcomes is essential, e.g., for a risk-averse decision maker, the P10 estimate of the project value is more important than the expected value. By performing multiple CLFD runs, results similar to those presented in Table 5 and Fig 16 can be obtained. These results present an assessment of the project outcome when optimal reservoir management is to be applied.

The TruMAP statistical assessment procedure can be applied to test and compare the effect of different treatments. Different geostatistical algorithms, optimization methods, representative model selection, or model calibration methods can be applied in closedloop optimization. In order to compare two different treatments, multiple runs should be performed and results can be analyzed in terms of a performance measure, e.g., average or median true-model improvement, or difference in probability of increasing true-model NPV.

The statistical performance assessment procedure (TruMAP) and results represent an example of massive computational experiments to quantify the effectiveness of a new algorithm. As Monajemi et al (2016) discussed, with advancement of computational power and tools, researchers are expected to present findings that involve such massive computations (order one million CPU-hours) as opposed to deducing conclusions from a limited number of test cases.

\section{Conclusions and future work}

In this work, a new implementation of closed-loop field development (CLFD) optimization is presented for models described by multipoint geostatistics (MPS). CLFD involves three major steps: 1) optimization of the full development plan for the entire project life, 2) drilling and completing $n_{\text {rig }}$ new wells and collecting new data, and 3) updating models based on all data. The optimization step is performed over multiple models through optimization 
with sample validation (OSV). A two-step model calibration procedure is presented where spatial data (hard data at well locations) are integrated through a MPS simulation method, and production data are integrated through a gradient-based RML procedure using O-PCA parameterization.

The new TruMAP procedure is introduced for performance assessment of a closed-loop optimization algorithm. Within this procedure, the new CLFD methodology is applied 25 times (for 25 different true-model cases), and the CLFD performance was assessed in terms of true NPV improvement. Results indicated that the true NPV in 64\%, 68\% and 96\% of cases increased after one, two and three steps, respectively. These results showed that application of multi-step CLFD is much more likely to increase true NPV than a single step CLFD, particularly as richer data appears at later CLFD steps. Further, the probability of increasing true-model NPV increases with CLFD step. The statistical assessment results presented in this work, demonstrates the importance of performing multiple runs and considering several true-model cases to assess the performance of any closed-loop optimization framework.

There are several directions to investigate in future. Application of CLFD optimization to geothermal reservoir, groundwater remediation (Bayer et al, 2008; Ghorbanidehno et al, 2017), and $\mathrm{CO}_{2}$-EOR problems, and unconventional resources (Shirangi et al, 2019) should also be investigated. In this work, a fixed project life was specified in the optimization step of CLFD and the expected NPV was optimized. It will be of interest to assess CLFD performance when the rate of return is incorporated in optimization objective and the economic project life is also optimized (Shirangi et al, 2017). New implementations of closedloop optimization should be statistically assessed by application of TruMAP procedure.

\section{Acknowledgments}

This research was conducted at Stanford University between 2015 and 2017. Access to the computational resources provided by the Stanford Center for Computational Earth \& Environmental Science (CEES) is gratefully acknowledged.

\section{References}

Aitokhuehi I, Durlofsky LJ (2005) Optimizing the performance of smart wells in complex reservoirs using continuously updated geological models. Journal of Petroleum Science and Engineering 48(3):254-264

D. Arnold, V. Demyanov, M. Christie, A. Bakay, and K. Gopa. Optimisation of decision making under uncertainty throughout field lifetime: A fractured reservoir example. Computers \& Geosciences, 95:123-139, 2016.

Barros EGD, Van den Hof PMJ, Jansen JD (2016) Value of information in closed-loop reservoir management. Computational Geosciences 20(3): 737-749. DOI https://doi.org/ $10.1007 / \mathrm{s} 10596-015-9509-4$

Bayer P, Finkel M (2007) Optimization of concentration control by evolution strategies: 
Formulation, application, and assessment of remedial solutions. Water resources research $43(2)$

Bayer P, Bürger C, Finkel M (2008) Computationally efficient stochastic optimization using multiple realizations. Advances in Water Resources 31(2):399-417

Bayer P, de Paly M, Bürger CM (2010) Optimization of high-reliability-based hydrological design problems by robust automatic sampling of critical model realizations. Water Resources Research 46(5)

Bellout MC, Ciaurri DE, Durlofsky LJ, Foss B, Kleppe J (2012) Joint optimization of oil well placement and controls. Computational Geosciences 16(4):1061-1079

Bjarkason EK, Maclaren OJ, O'Sullivan JP, O'Sullivan MJ, (2017) Randomized Truncated SVD Levenberg-Marquardt Approach to Geothermal Natural State and History Matching. preprint arXiv:1710.01031.

Bickel JE, Bratvold RB (2008) From uncertainty quantification to decision making in the oil and gas industry. Energy Exploration \& Exploitation 26(5): 311-325

Bouzarkouna Z, Ding DY, Auger A (2012) Well placement optimization with the covariance matrix adaptation evolution strategy and meta-models. Computational Geosciences 16(1):75-92

Bukshtynov V, Volkov O, Durlofsky LJ, Aziz K (2015) Comprehensive framework for gradient-based optimization in closed-loop reservoir management. Computational Geosciences 19(4):877-897

J. Caers. History matching under training-image-based geological model constraints. SPE Journal, 8(3):218-226, 2003.

D. A. Cameron and L. J. Durlofsky. Optimization and data assimilation for geological carbon storage. In: R. Al-Khoury, J. Bundschuh (Eds.), Computational Models for $\mathrm{CO}_{2}$ Geo-sequestration \& Compressed Air Energy Storage. CRC Press, 355-388, 2014.

Cameron DA, Durlofsky LJ (2012) Optimization of well placement, $\mathrm{CO}_{2}$ injection rates, and brine cycling for geological carbon sequestration. International Journal of Greenhouse Gas Control 10:100-112

Chen Y, Oliver DS, Zhang D, et al (2009) Efficient ensemble-based closed-loop production optimization. SPE Journal 14(04):634-645

Dickstein F, Goldfeld P, Pfeiffer GT, Pinto RV (2017) Truncated conjugate gradient and improved LBFGS and TSVD for history matching. Computational Geosciences, DOI https://doi.org/10.1007/s10596-017-9694-4

Ghorbanidehno H, Kokkinaki A, Li JY, Darve E, Kitanidis PK (2015) Real-time data assimilation for large-scale systems: The spectral Kalman filter. Advances in Water Resources, $86: 260-272$. 
Ghorbanidehno H, Kokkinaki A, Kitanidis PK, Darve E (2017) Optimal estimation and scheduling in aquifer management using the Rapid Feedback Control Method. Advances in Water Resources, DOI https://doi.org/10.1016/j.advwatres.2017.10.011

Ghorbanidehno H (2018) Fast Data Assimilation and Optimal Control Methods and Applications. PhD dissertation, Stanford University.

Gill PE, Murray W, Saunders MA (2005) SNOPT: An SQP algorithm for large-scale constrained optimization. SIAM review 47(1):99-131

Hanea RG, Casanova P, Hustoft L, Bratvold RB, et al (2017) Drill and Learn: A Decision Making Workflow to Quantify Value of Learning. Paper SPE-182719-MS presented at SPE Reservoir Simulation Conference, Houston, Texas, DOI https://doi.org/10.2118/ 182719-MS

Hidalgo DM, Emerick AA, Couto P, et al (2017) Closed-loop field development under geological uncertainties: application in a Brazilian benchmark case. Paper OTC-28089-MS presented at Offshore Technology Conference, Rio de Janeiro, Brasil.

Howard, R A (1988) Decision analysis: practice and promise. Management science 34(6):679-695 DOI https://doi.org/10.1287/mnsc.34.6.679

Isebor OJ, Durlofsky LJ, Ciaurri DE (2014a) A derivative-free methodology with local and global search for the constrained joint optimization of well locations and controls. Computational Geosciences (18):463-482

Isebor OJ, Echeverría Ciaurri D, Durlofsky LJ (2014b) Generalized field-development optimization with derivative-free procedures. SPE Journal (19):891-908

Jansen JD, Brouwer DR, Naevdal G, van Kruijsdijk CPJW (2005) Closed-loop reservoir management. First Break, January, 23, 4348.

Jansen JD, Douma SD, Brouwer DR, den Hof PMJV, et al (2009) Closed-loop reservoir management. Paper SPE 119098 presented at SPE Reservoir Simulation Symposium, The Woodlands, Texas.

Kitanidis PK (1995) Quasi-linear geostatistical theory for inversing. Water Resources Research 31(10):2411-2419

Li L, Jafarpour B (2012) A variable-control well placement optimization for improved reservoir development. Computational Geosciences 16(4):871-889

Liu Y (2017) Multilevel Strategy for O-PCA-based History Matching Using Mesh Adaptive Direct Search. MS thesis, Stanford University.

Mariethoz G, Caers J (2014) Multiple-Point Geostatistics: Stochastic Modeling with Training Images. John Wiley \& Sons.

Monajemi H, Donoho DL, Stodden V (2016) Making massive computational experiments painless. IEEE International Conference on Big Data. 
Morosov AL, Schiozer DJ (2016) Field development process revealing uncertainty assessment pitfalls. Paper SPE-180094-MS presented at the SPE Europec featured at 78th EAGE Conference, Vienna, Austria. DOI https://doi.org/10.2118/180094-MS

Nwankwor E, Nagar AK, Reid D (2013) Hybrid differential evolution and particle swarm optimization for optimal well placement. Computational Geosciences 17(2):249-268

Onwunalu JE, Durlofsky L (2010) Application of a particle swarm optimization algorithm for determining optimum well location and type. Computational Geosciences 14(1): 183198.

Ozdogan U, Horne RN (2006) Optimization of well placement under time-dependent uncertainty. SPE Journal 9(2):135-145

Rafiee J (2017) Data Assimilation and Uncertainty Quantification Using the EnsembleBased Methods with and Without Markov Chain Monte Carlo. PhD thesis, University of Tulsa.

Rafiee J, Reynolds AC (2017) Theoretical and efficient practical procedures for the generation of inflation factors for ES-MDA. Inverse Problems 33(11):115003

Rafiee J, Reynolds AC (2018) A two-level MCMC based On the distributed Gauss-Newton method for uncertainty quantification. Proceedings of the 16th European Conference on the Mathematics of Oil Recovery.

Regtien JM. Extending the smart fields concept to enhanced oil recovery. Paper SPE 136034 presented at SPE Russian Oil and Gas Conference, Moscow, Russia, 2010.

Shirangi MG (2011) History Matching Production Data with Truncated SVD Parameterization. Master's thesis, University of Tulsa.

Shirangi MG, Mukerji T (2012) Retrospective optimization of well controls under uncertainty using kernel clustering. Proceedings of the 25th Annual Meeting of the Stanford Center for Reservoir Forecasting, Monterey, California.

Shirangi MG (2013) Closed-loop field development optimization. Proceedings of the 26th Annual Meeting of Stanford Center for Reservoir Forecasting, Palo Alto, California.

Shirangi MG (2014) History matching production data and uncertainty assessment with an efficinet TSVD parameterization algorithm. Journal of Petroleum Science and Engineering 113:54-71, DOI https://doi.org/10.1016/j.petrol.2013.11.025

Shirangi MG, Durlofsky LJ (2015) Closed-loop field development under uncertainty by use of optimization with sample validation. SPE Journal 20(05):908 - 922, DOI https: //doi.org/10.2118/173219-PA

Shirangi MG, Durlofsky LJ (2016) A general method to select representative models for decision making and optimization under uncertainty. Computers \& Geosciences 96:109123. DOI https://doi.org/10.1016/j.cageo.2016.08.002 
Shirangi MG, Emerick AA (2016) An improved TSVD-based Levenberg-Marquardt algorithm for history matching and comparison with Gauss-Newton. Journal of Petroleum Science and Engineering 143:258-271, DOI https://doi.org/10.1016/j.petrol.2016.02.026

Shirangi MG (2017) Advanced Techniques for Closed-Loop Reservoir Optimization under Uncertainty. PhD Thesis, Stanford University.

Shirangi MG, Volkov O, Durlofsky LJ (2017) Joint optimization of economic project life and well controls. SPE Journal, DOI https://doi.org/10.2118/182642-PA

Shirangi MG, Oruganti Y, Wilson TA, Furlong E et al (2019) Prescriptive analytics for completion optimization in unconventional resources. Paper SPE-195311-MS presented at SPE Western Regional Meeting, San Jose, California, DOI https://doi.org/10.2118/ 195311-MS

Simmons, JP, Nelson, LD, Simonsohn, U (2011) False-positive psychology: Undisclosed flexibility in data collection and analysis allows presenting anything as significant. Psychological Science, 22(11):1359-1366, DOI https://doi.org/10.1177/0956797611417632

Tahmasebi P, Sahimi M, Caers J (2014) MS-CCSIM: accelerating pattern-based geostatistical simulation of categorical variables using a multi-scale search in Fourier space. Computers \& Geosciences 67:75-88

Tarantola A (2005) Inverse Problem Theory and Methods for Model Parameter Estimation. SIAM, Philadelphia, USA

Vo HX, Durlofsky LJ (2014) A new differentiable parameterization based on principal component analysis for the low-dimensional representation of complex geological models. Mathematical Geosciences 46(7):775-813

Vo HX, Durlofsky LJ (2015) Data assimilation and uncertainty assessment for complex geological models using a new PCA-based parameterization. Computational Geosciences 19(4):747-767

Vo HX, Durlofsky LJ (2016) Regularized kernel PCA for the efficient parameterization of complex geological models. Journal of Computational Physics 322:859-881, DOI https: //doi.org/10.1016/j.jcp.2016.07.011

Wilson KC, Durlofsky LJ (2013) Optimization of shale gas field development using direct search techniques and reduced-physics models. Journal of Petroleum Science and Engineering 108:304-315

Younis MR (2011) Modern Advances in Software and Solution Algorithms for Reservoir Simulation. Ph.D. thesis, Stanford University.

Zhang Y, Lu R, Forouzanfar F, Reynolds AC (2017) Well placement and control optimization for WAG/SAG processes using ensemble-based method. Computers \& Chemical Engineering 101: 193-209 
Zhou Y, Tchelepi H (2012) Multi-core and GPU parallelization of a general purpose reservoir simulator. Proceedings of the 13th European Conference on the Mathematics of Oil Recovery, Biarritz, France. 